\title{
Reconstitution of bacterial autotransporter assembly using purified components
}

\author{
Giselle Roman-Hernandez, Janine H Peterson, Harris D Bernstein*
}

Genetics and Biochemistry Branch, National Institute of Diabetes and Digestive and Kidney Diseases, National Institutes of Health, Bethesda, United States

\begin{abstract}
Autotransporters are a superfamily of bacterial virulence factors consisting of an $N$-terminal extracellular ('passenger') domain and a C-terminal $\beta$ barrel (' $\beta$ ') domain that resides in the outer membrane (OM). The mechanism by which the passenger domain is secreted is poorly understood. Here we show that a conserved OM protein insertase (the Bam complex) and a molecular chaperone (SurA) are both necessary and sufficient to promote the complete assembly of the Escherichia coli O157:H7 autotransporter EspP in vitro. Our results indicate that the membrane integration of the $\beta$ domain is the rate-limiting step in autotransporter assembly and that passenger domain translocation does not require the input of external energy. Furthermore, experiments using nanodiscs strongly suggest that autotransporter assembly is catalyzed by a single copy of the Bam complex. Finally, we describe a method to purify a highly active form of the Bam complex that should facilitate the elucidation of its function.

DOI: 10.7554/eLife.04234.001
\end{abstract}

*For correspondence: harris_ bernstein@nih.gov

Competing interests: The authors declare that no competing interests exist.

Funding: See page 18

Received: 02 August 2014

Accepted: 01 September 2014

Published: 02 September 2014

Reviewing editor: Ramanujan S Hegde, MRC Laboratory of Molecular Biology, United Kingdom

(c) This is an open-access article, free of all copyright, and may be freely reproduced, distributed, transmitted, modified, built upon, or otherwise used by anyone for any lawful purpose. The work is made available under the Creative Commons CCO public domain dedication.

\section{Introduction}

The autotransporter (type $\mathrm{Va}$ ) pathway is the most widespread virulence factor secretion pathway in Gram-negative bacteria (Leyton et al., 2012). Autotransporters are single polypeptides that consist of two domains, an N-terminal domain ('passenger domain') that is exposed on the cell surface and that often mediates a virulence function, and a C-terminal domain (' $\beta$ domain') that resides in the outer membrane $(\mathrm{OM})$. Following their translocation across the $\mathrm{OM}$, many passenger domains are released from the cell surface by a proteolytic cleavage. Passenger domains vary widely in sequence and size $(\sim 20-400 \mathrm{kD}$ ), but almost always form an unusual repetitive structure known as a $\beta$ helix (Junker et al., 2006). $\beta$ domains are typically $\sim 30 \mathrm{kD}$ in size and, like the vast majority of bacterial integral OM proteins, fold into a $\beta$ barrel structure. While the sequences of $\beta$ domains are also very heterogeneous, the structures of all of the $\beta$ domains that have been solved to date are nearly superimposable (Oomen et al., 2004; Barnard et al., 2007; van den Berg, 2010; Zhai et al., 2011). After autotransporters are translocated across the inner membrane (IM) through the Sec complex they interact with molecular chaperones that presumably maintain them in an assembly-competent state (leva and Bernstein, 2009; Ruiz-Perez et al., 2009; leva et al., 2011). Subsequently the $\beta$ domain is targeted to the Bam complex, an essential heterooligomer consisting of an integral OM protein (BamA) and four lipoproteins (BamBCDE) that catalyzes the membrane insertion of $\beta$ barrel proteins (Voulhoux et al., 2003; Wu et al., 2005; Sauri et al., 2009; Hagan et al., 2010; leva et al., 2011). X-ray crystallographic analysis suggests that the BamA $\beta$ barrel domain is unstable and may open laterally to allow the escape of client proteins into the lipid bilayer (Noinaj et al., 2013, 2014).

Although it has been shown that the passenger domain is translocated across the OM in a C-to-Nterminal fashion (leva and Bernstein, 2009; Junker et al., 2009), the mechanism of translocation is poorly understood. Based on the observation that deletion of the $\beta$ domain abolishes passenger domain translocation, it was originally proposed that the $\beta$ domain serves as the transport channel for the passenger domain (whence the name 'autotransporter') (Pohlner et alo, 1987). At first glance, this 
eLife digest Disease-causing bacteria release molecules called virulence factors to help them infect their host. These virulence factors need to pass through the membrane that surrounds the cell. Indeed, some bacteria, such as Escherichia coli, have two membranes, so some virulence factors need to pass through an extra membrane.

One group of virulence factors found in E. coli are called autotransporters. These proteins have two sections: the passenger domain, which is the main part of the virulence factor, and the $\beta$ domain, which anchors the autotransporter in the outer membrane. Once the passenger domain is outside the cell, the link to the $\beta$ domain can be broken to release the virulence factor. However, we do not know how the passenger domain passes through the outer membrane.

By studying an E. coli autotransporter called EspP, Roman-Hernandez et al. have now identified the other proteins that are required for the $\beta$ domain to insert into an artificial membrane, and allow the passenger domain to pass through the membrane. These other proteins are a group of proteins called the Bam complex and a chaperone protein called SurA. The experiments also show that an external source of energy is not needed to drive this process, and they suggest that the passenger domain moves through a hole in the outer membrane formed by the $\beta$ domain and/or the Bam complex. Roman-Hernandez et al. also developed a new way to purify the Bam complex that should help all researchers working on this set of proteins.

DOI: 10.7554/eLife.04234.002

hypothesis seems to be supported by crystallographic studies showing that upon completion of translocation the two domains are connected by an $\alpha$-helical linker that is embedded inside the $\beta$ domain pore (Oomen et al., 2004; Barnard et al., 2007; van den Berg, 2010). The same studies, however, revealed that the $\beta$ domain pore is only $\sim 10 \AA$ in diameter. Because the directionality of translocation presumably requires the formation of a $\mathrm{C}$-terminal hairpin followed by the sliding of medial and $\mathrm{N}$-terminal segments past a static strand, both strands of the hairpin would have to be in a fully extended conformation to fit inside the pore. On the contrary, available evidence indicates that the polypeptide that resides inside the $\beta$ domain forms an $\alpha$ helix prior to the completion of translocation (leva et al., 2008; Peterson et al., 2010). Furthermore, small folded polypeptides have been shown to be secreted efficiently by the autotransporter pathway and a subset of naturally occurring passenger domains undergo disulfide bonding in the periplasm (Skillman et al., 2005; Jong et al., 2007). Finally, BamA has been shown to interact with the passenger domain during the translocation reaction (leva and Bernstein, 2009). While the $\beta$ domain does appear to play a role in translocation (Saurí et al., 2011; Pavlova et al., 2013), these and other results strongly suggest that autotransporter assembly is more complex than originally envisioned. In one scenario, the Bam complex might facilitate the membrane insertion of the $\beta$ domain and the translocation of the passenger domain in a concerted reaction, but it is also possible that other factors are also required to transport the passenger domain across the OM.

The energy source for passenger domain translocation has also remained poorly understood. Although most protein translocation reactions require external energy in the form of ATP or a membrane potential, the periplasm is devoid of ATP and there is no membrane potential across the bacterial OM. It is conceivable that an IM protein that derives energy from the hydrolysis of cytoplasmic ATP or from the electrochemical gradient across the IM interacts with the passenger domain and drives translocation, but such a protein has never been identified. To explain the energetics of secretion, it has been proposed that the sequential folding of segments of the $\beta$ helix in the extracellular space drives translocation and prevents retrograde movement into the periplasm (Klauser et alo, 1992; Junker et al., 2006). Consistent with this vectorial folding model, mutations that impair the folding of $\mathrm{C}$-terminal segments of two different passenger domains significantly reduce the efficiency of secretion (Peterson et al., 2010; Renn et al., 2012). Mutations that perturb the folding of medial and $\mathrm{N}$-terminal segments of the passenger domain, however, produce only a modest translocation defect (Kang'ethe and Bernstein, 2013b). Furthermore, the intrinsically disordered receptor domain (RD) of the Bordetella pertussis RTX toxin has been shown to be secreted efficiently by the autotransporter pathway (Kang'ethe and Bernstein, 2013a). Interestingly, the presence of a large number of acidic 
residues is critical for the secretion of the RD. This observation, together with the finding that naturally occurring passenger domains are predominantly acidic, suggests that charge interactions may play a significant role in driving the translocation reaction.

To gain further insight into both the mechanism and energetics of passenger domain translocation, we sought to reconstitute autotransporter assembly in vitro using purified components. In our experiments, we used the Escherichia coli 0157:H7 autotransporter EspP, as a model protein. Following the completion of translocation, the passenger domain of EspP and other members of the SPATE (serine protease autotransporters of Enterobacteriaceae) family is released in an unusual intrabarrel cleavage reaction that requires accurate folding of the $\beta$ domain and precise positioning of the passenger domain- $\beta$ domain junction (Dautin et al., 2007; Barnard et al., 2012). Although it was previously shown that the insertion of small E. coli $\beta$ barrel proteins such as OmpT and OmpA into proteoliposomes can be catalyzed by the purified Bam complex and the periplasmic chaperone SurA (Hagan et al., 2010; Hagan and Kahne, 2011; Hagan et al., 2013), we did not observe assembly of EspP using the same methodology. Using an alternative expression and purification strategy, however, we obtained an apparently more active form of the Bam complex that, together with SurA, was both necessary and sufficient to promote the membrane integration of the EspP $\beta$ domain and the translocation and cleavage of the EspP passenger domain. Remarkably, passenger domain translocation did not require the input of any additional energy. In addition to defining the minimal set of factors required for autotransporter assembly, our work provides a valuable resource for future studies on the function of the Bam complex and its role in the biogenesis of the broader class of OM proteins.

\section{Results}

\section{The Bam complex and SurA are necessary and sufficient to promote the assembly of an EspP derivative that contains a minimal passenger domain fragment}

It has long been known that large N-terminal segments of autotransporter passenger domains can be deleted without affecting the integration of the $\beta$ domain into the $\mathrm{OM}$ or the secretion of the remaining passenger domain fragment (Dautin and Bernstein, 2007). The native EspP passenger domain is 968 residues in length (Figure 1A), but a derivative that contains only 26 residues of the $\sim 28$ residue $\mathrm{C}$-terminal segment that reside inside the $\beta$ domain in an $\alpha$-helical conformation $(E s p P \Delta 5)$ is assembled as efficiently as the wild-type protein in vivo (Pavlova et al., 2013). The modification or deletion of residues in the a-helical segment, however, can profoundly perturb the folding and integration of the $\beta$ domain into the $O M$ and/or the proteolytic release of the passenger domain, which requires precise alignment of the cleavage junction with key catalytic residues (Dautin et al., 2007; leva et al., 2008; Barnard et al., 2012). Because EspP $\Delta 5$ and other derivatives that expose no more than a short segment on the cell surface are structurally similar to generic $\beta$ barrel proteins such as OmpT, it might be expected that they would have similar assembly requirements. Indeed any autotransporter-specific assembly factors might only be required once the passenger domain reaches a threshold size. Based on this reasoning, we first analyzed the assembly of an EspP derivative designated $\operatorname{EspP}(46+\beta)$ that consists of EspP $\Delta 5$ plus $20 \mathrm{~N}$-terminal residues derived from the cloning vector (Figure $1 B$ ). We expected that the accurate assembly of EspP $(46+\beta)$ would result in the proteolytic release of the 46 residue passenger domain and the accumulation of a folded $\sim 30 \mathrm{kD} \beta$ domain that, as previously shown, is resistant to SDS denaturation unless heated (Skillman et al., 2005).

Initially we tested whether $\operatorname{EspP}(46+\beta)$ would assemble in vitro using a previously described OM protein assembly assay (Hagan et al., 2010). In this assay, E. coli BamAB and BamCDE are first expressed and isolated independently. The two subcomplexes are then mixed together to form a holocomplex and reconstituted into proteoliposomes. In the presence of the proteoliposomes and a molar excess of SurA, denatured OmpT folds into a stable structure that is enzymatically active and resistant to SDS denaturation. We attempted to express and purify the Bam complex exactly as described, but for reasons that are unclear we did not observe efficient formation of the Bam holocomplex unless we purified BamAB through an additional step. Nevertheless, we ultimately obtained a single peak on a gel filtration column that was highly enriched in the reconstructed complex, which we designated $\operatorname{Bam}(A B)(C D E)$ (Figure 2-figure supplement 1A,B). After the peak fractions were pooled, 


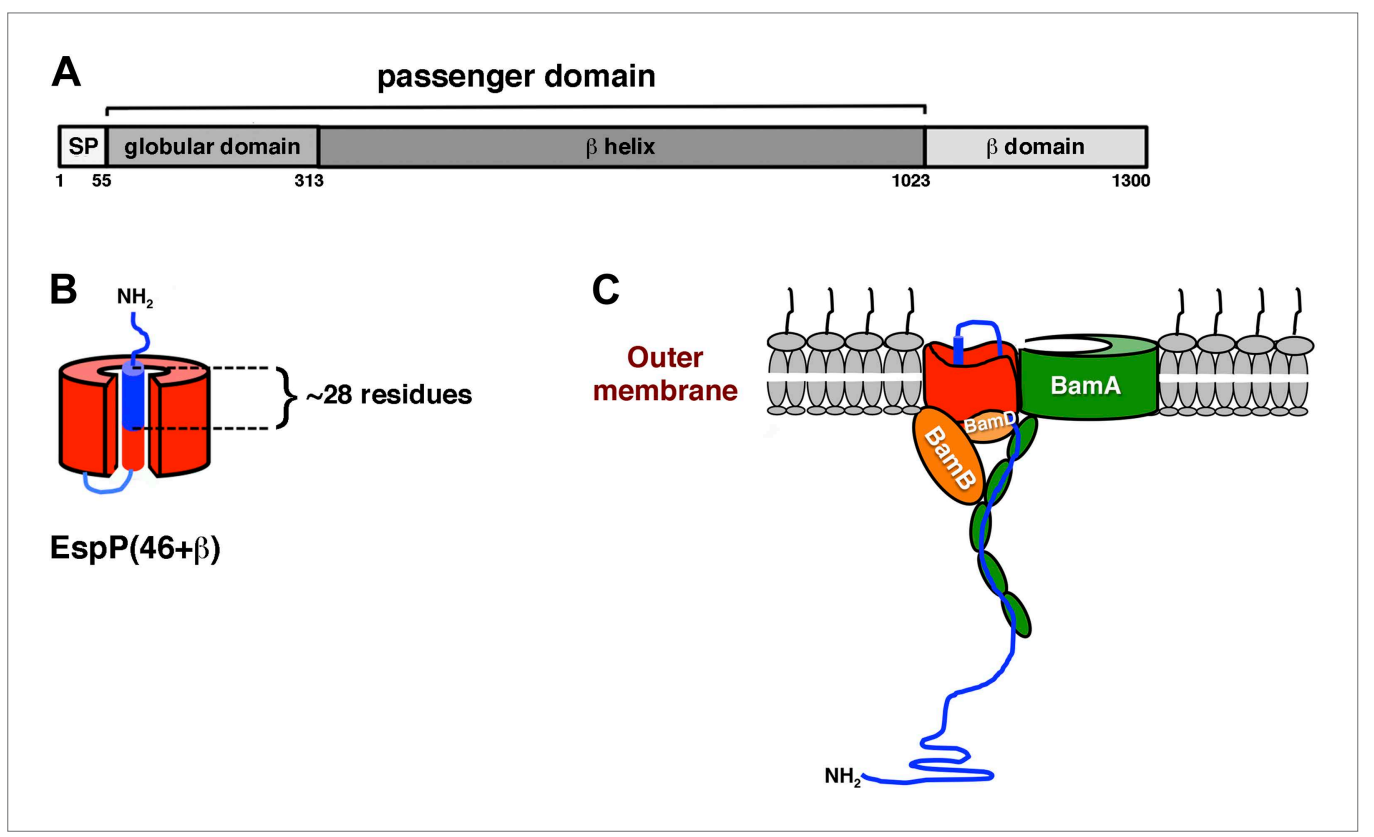

Figure 1. Domain structure of EspP and model for passenger domain translocation. (A) EspP consists of a signal peptide (SP; residues 1-55), an extracellular ('passenger') domain (residues 56-1023) and a $\beta$ barrel (' $\beta$ ') domain (residues 1024-1300) (Brunder et al., 1997). While most of the passenger domain forms a long $\beta$ helix, the $\mathrm{N}$-terminus (residues 56-313) forms a discrete globular domain (Khan et al., 2011). (B) Illustration of EspP(46+ $\beta$ ). Prior to the release of the passenger domain in an intrabarrel cleavage reaction, $\sim 28$ residues of the 46 residue passenger domain are embedded inside the $\beta$ domain pore. (C) Available evidence indicates that the EspP passenger domain is secreted in a hairpin conformation while distinct regions of the $\beta$ domain interact with BamA, BamB and BamD (leva et al., 2011). Components of the transport channel likely include the open $\beta$ domain and/ or the BamA $\beta$ barrel, which has been proposed to open laterally (Noinaj et al., 2013, 2014). In any case, the finding that proteolytic maturation and the release of the $\beta$ domain from the Bam complex both require the completion of translocation (Peterson et al., 2010; leva et al., 2011; Pavlova et al., 2013) strongly suggests that the active site cannot form during the passage of the passenger domain across the OM. BamC and BamE hve been omitted from the model for the sake of clarity.

DOI: 10.7554/eLife.04234.003

most of the Bam holocomplex could be reconstituted into proteoliposomes (Figure 2-figure supplement 1C). Subsequently we purified SurA to homogeneity as described (Hagan et al., 2010) (Figure 2-figure supplement 2). To assess the folding of OmpT, we used an activity assay in which the cleavage of a fluorogenic peptide leads to an increase in fluorescence intensity over time. Consistent with previous results, we observed a fluorescent signal when OmpT was incubated in the presence of $\operatorname{Bam}(A B)(C D E)$ and SurA (Figure 2E, purple curve). EspP(46+ $\beta$ ) purified from inclusion bodies (Figure 3-figure supplement 1) was mixed with the same components, and the proteolytic maturation of the protein was assessed by Western blotting using an antiserum against an EspP C-terminal peptide. No free $\beta$ domain was detected, however, even after a prolonged incubation (Figure 2-figure supplement 1D).

Partly because we had difficulty reconstructing the Bam complex from BamAB and BamCDE subcomplexes, we next cloned the genes encoding all five subunits into a single expression plasmid. An octahistidine tag was introduced at the C-terminus of BamE to facilitate Bam complex purification. All of the proteins co-eluted on gel filtration columns (Figure 2A,B). This observation strongly suggests that Bam holocomplexes were formed efficiently in vivo. When the peak fractions were pooled and analyzed by Blue Native PAGE, a single band that migrated slightly slower than the 242 kDa marker was seen (Figure 2C). The Bam complex is only $200 \mathrm{kDa}$, but it has previously been shown to migrate slower than its actual molecular weight on Blue Native gels (Hagan et al., 2013). In the presence of E. coli phospolipids, almost all of the purified protein, which we designated BamABCDE, could be reconstituted into proteoliposomes (Figure 2D). Remarkably, the intensity of the fluorescent signal in the fluorogenic peptide cleavage assay indicated that OmpT folded much more efficiently in the 
A

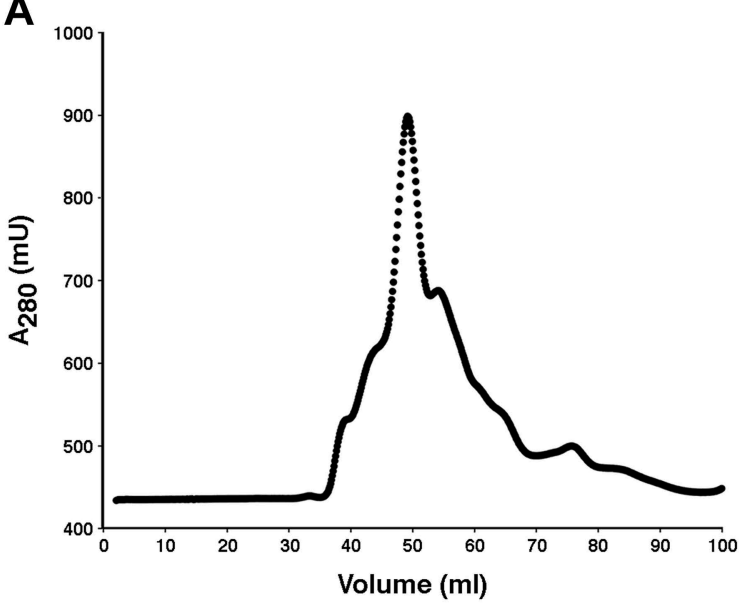

\section{B}

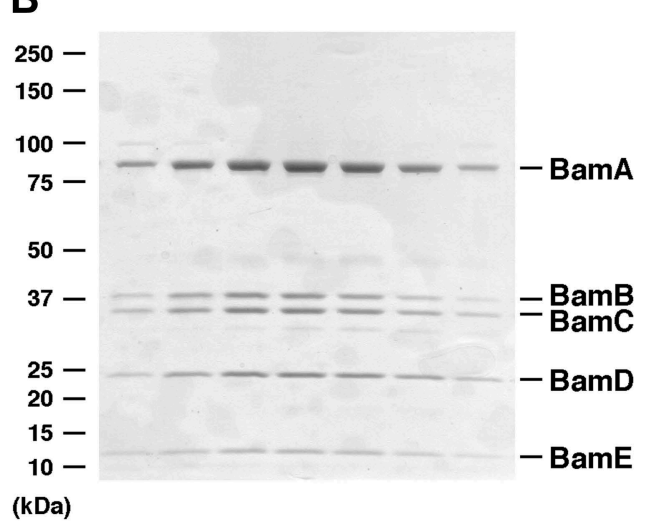

C

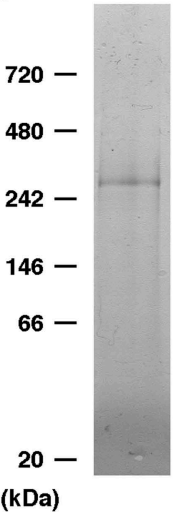

D

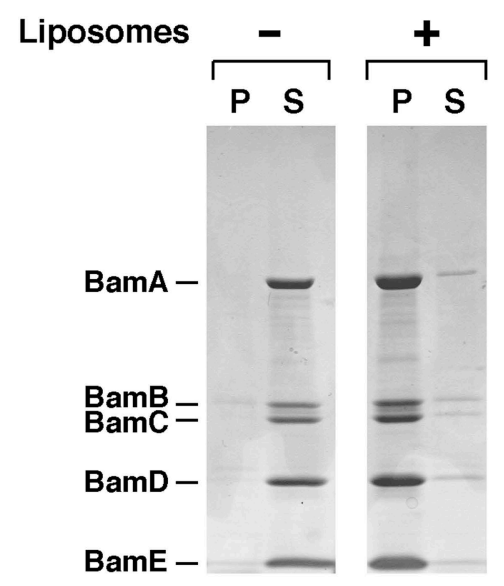

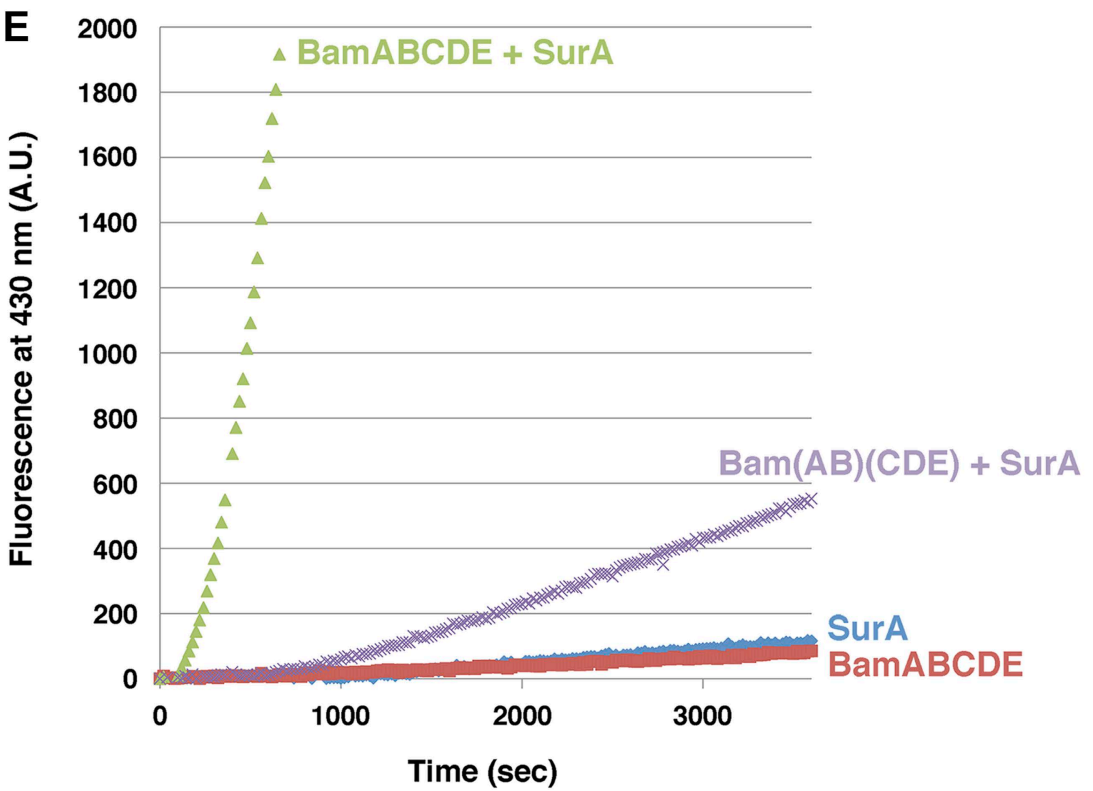

Figure 2. Purification and functional test of BamABCDE. (A) Chromatogram of BamABCDE on S-200 gel filtration column. (B) SDS-PAGE analysis of the peak fractions in (A). Proteins were visualized by Coomassie Blue staining. (C) The peak fractions in (B) were pooled and analyzed by Blue Native PAGE. (D) Purified BamABCDE was centrifuged in the absence of liposomes or after reconstitution into liposomes. The pellet (P) and supernatant (S) fractions were analyzed by SDS-PAGE. (E) Urea denatured OmpT was diluted and incubated with SurA and proteoliposomes containing either BamABCDE (green) or Bam(AB)(CDE) (purple), proteoliposomes containing BamABCDE alone (red) or SurA alone (blue). OmpT activity was assessed by measuring the fluorescent signal generated by the cleavage of a fluorogenic peptide.

DOI: $10.7554 /$ LLife.04234.004

The following figure supplements are available for figure 2:

Figure supplement 1. Test of purified Bam(AB)(CDE) in EspP assembly assay. DOI: 10.7554/eLife.04234.005

Figure supplement 2. SDS-PAGE analysis of purified SurA.

DOI: 10.7554/eLife.04234.006

presence of BamABCDE than $\operatorname{Bam}(A B)(C D E)$ (Figure 2E, compare green and purple curves). These results suggested that the activity of the Bam complex is optimized when all of the subunits are expressed together.

Interestingly, we found that BamABCDE also stimulated the assembly of EspP $(46+\beta)$. The ureadenatured EspP derivative was incubated at $30^{\circ} \mathrm{C}$ with proteoliposomes containing the Bam complex 
and SurA, and samples were removed at various time points. Folding of the protein and subsequent proteolytic maturation were monitored by Western blot using the anti-EspP C-terminal antiserum and a fluorescently-labeled secondary antibody. A rapidly migrating ( 27 kD) band that corresponds to the folded form of the free EspP $\beta$ domain appeared within 5 min and was more prominent at later time points when the samples were not heated (Figure 3A). When the samples were heated, a much more intense $\sim 30 \mathrm{kD}$ band that corresponds to the unfolded $\beta$ domain was observed. This marked difference in intensity has been observed previously (Barnard et al., 2007; Pavlova et al., 2013) and presumably results from the occlusion of the $C$-terminal epitope in the folded form of the $\beta$ domain. No proteolytic processing was observed if either SurA or BamABCDE was omitted from the reaction or if BamAB or BamCDE subomplexes were used in place of the holocomplex (Figure 3B). Single plasmids were also used to produce Bam complexes that lack either BamB or BamC, but curiously only $50-70 \%$ of the purified partial complexes could be reconstituted into proteoliposomes (Figure 3-figure supplement $2 A)$. BamABDE and BamACDE both promoted the assembly $\operatorname{Esp} P(46+\beta)$, but less effectively than BamABCDE (Figure 3-figure supplement 2B). Finally, the addition of purified Skp to reactions containing BamABCDE and SurA inhibited $\operatorname{EspP}(46+\beta)$ assembly (Figure 3-figure supplement 3) and ATP $(0.1 \mathrm{mM})$ blocked assembly altogether (data not shown).

To confirm that the $\sim 30 \mathrm{kDa}$ fragment we observed was identical to the polypeptide that results from proteolytic maturation in vivo, we examined the assembly of a non-cleavable version of $\operatorname{EspP}(46+\beta)$. This derivative, which is designated $\operatorname{Esp} P^{*}(46+\beta)$, contains a mutation at the cleavage site that abolishes proteolysis but does not affect folding (Skillman et al., 2005). A polypeptide that migrated slower than the folded $\beta$ domain $(\sim 30 \mathrm{kDa}$ vs $\sim 27 \mathrm{kDa})$ and that corresponds to the folded form of EspP ${ }^{\star}(46+\beta)$ was observed when the samples were not heated (Figure $3 C$ ). As expected, this band disappeared when the samples were heated because in the absence of cleavage the denatured form of $\operatorname{Esp}^{*}(46+\beta)$ co-migrates with the unassembled protein.

Quantitation of the relative fluorescent signal produced by the denatured form of the EspP $\beta$ domain and unprocessed $\operatorname{EspP}(46+\beta)$ on Western blots indicated that $\sim 10-20 \%$ of the protein was typically assembled under our experimental conditions. The efficiency of assembly did not appear to be limited by the concentration of either reconstituted BamABCDE or SurA. A moderate increase in efficiency was observed when the BamABCDE concentration was increased from $0.05 \mu \mathrm{M}$ to $0.2 \mu \mathrm{M}$ (the concentration used in the experiments described here), but no increase was observed at higher concentrations (Figure 3-figure supplement 4A). Interestingly, if we added fresh substrate $5^{\prime}$ after the start of the incubation, the total yield of assembled $\operatorname{EspP}(46+\beta)$ increased in proportion to the amount of added substrate (Figure 3-figure supplement 4B). This observation is consistent with other evidence that the Bam complex can catalyze multiple rounds of assembly in vitro (Hagan and Kahne, 2011). In light of our results, it is likely that the efficiency of $\operatorname{EspP}(46+\beta)$ assembly was limited by the inherent ability of the denatured protein to remain assembly competent after dilution out of $8 \mathrm{M}$ urea.

\section{The Bam complex and SurA catalyze the assembly of larger EspP derivatives}

We next wished to determine whether the factors that promote the assembly of the EspP $\beta$ domain can also promote the translocation of substantial passenger domain fragments. To this end, we expressed and purified EspP derivatives that contain progressively longer portions of the native passenger domain plus $20 \mathrm{~N}$-terminal residues derived from the cloning vector (Figure 3-figure supplement 1). Because proteolytic maturation of EspP is absolutely dependent on the completion of passenger domain translocation in vivo (leva and Bernstein, 2009; Peterson et al., 2010; Pavlova et al., 2013), we initially assessed translocation by monitoring the appearance of the cleaved $\beta$ domain. Indeed available evidence strongly suggests that the completion of $\beta$ domain assembly (and the assembly of the active site) is a late event in autotransporter biogenesis (leva et al., 2011), possibly because the $\beta$ domain forms at least part of the passenger domain transport channel (Figure 1C). As Western blot analysis using the anti-EspP C-terminal antiserum indicated, EspP derivatives that have passenger domains ranging in size from 72-734 residues were all processed with approximately the same efficiency as EspP(46+ $\beta$ ) (Figure 4). The largest derivative lacks the $\mathrm{N}$-terminal globular segment of the EspP passenger domain, but contains the entire $\beta$ helical segment (Figure 1A). These results not only suggest that BamABCDE and SurA are sufficient to promote passenger domain translocation, but also suggest that the passenger domain does not readily adopt a translocation-incompetent conformation that independently limits the efficiency of autotransporter assembly. 


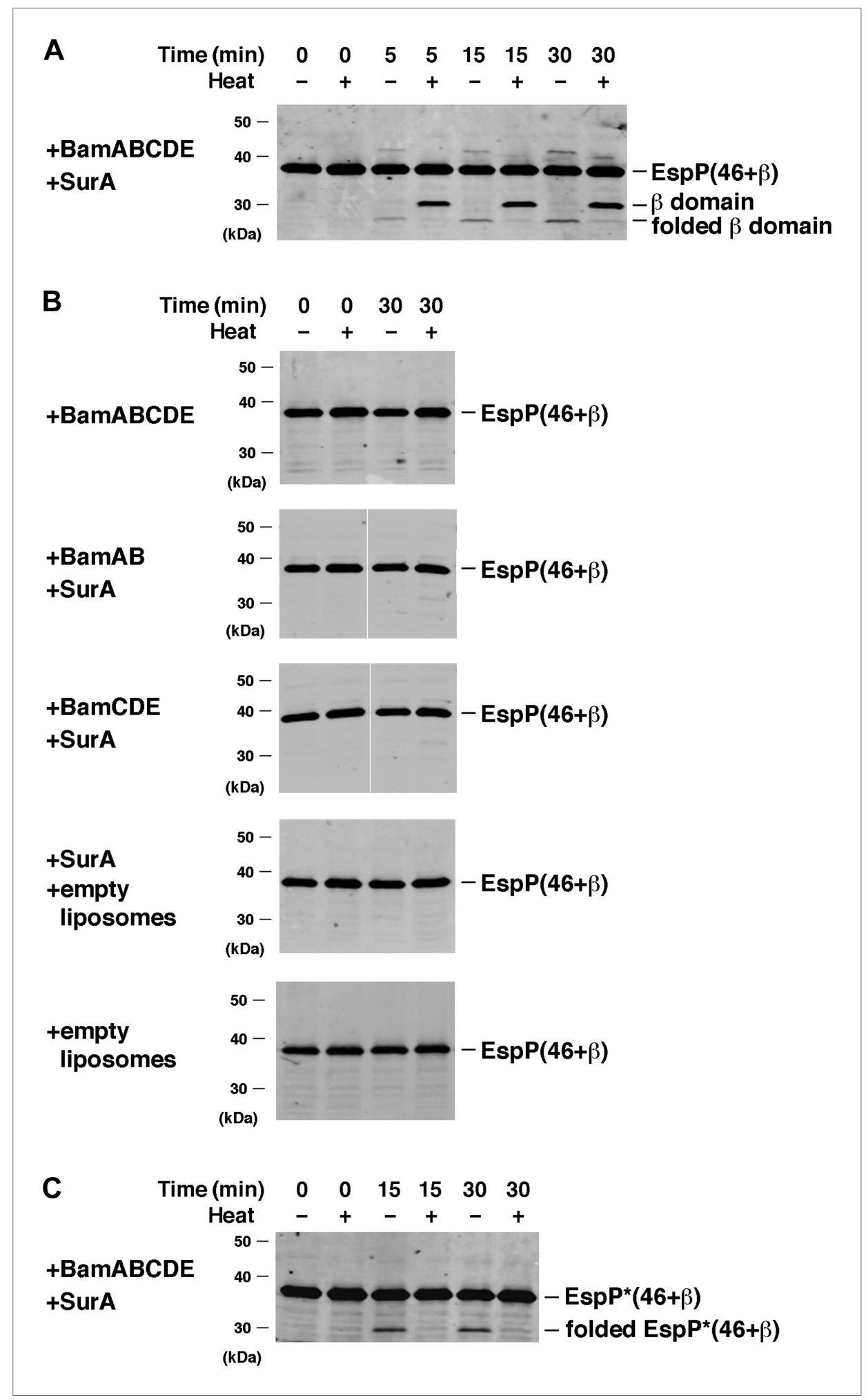

Figure 3. BamABCDE and SurA catalyze the assembly of $\operatorname{EspP}(46+\beta)$. (A) Urea-denatured $\operatorname{Esp} P(46+\beta)$ was incubated with SurA and proteoliposomes containing BamABCDE. Aliquots were placed on ice at various time points, heated to $95^{\circ} \mathrm{C}$ or maintained at room temperature after the addition of SDS-PAGE buffer, and analyzed by Western blot using an anti-EspP C-terminal peptide antiserum. (B) Urea-denatured EspP(46+ $\beta$ ) was incubated with the indicated factors. Aliquots were removed after 0 and $30 \mathrm{~min}$ and analyzed as in (A). (C) Urea-denatured Figure 3. Continued on next page 
Figure 3. Continued

Esp $P^{\star}(46+\beta)$ was diluted and incubated with SurA and proteoliposomes containing BamABCDE. Aliquots were removed at various time points and analyzed as in (A).

DOI: 10.7554/eLife.04234.007

The following figure supplements are available for figure 3:

Figure supplement 1. SDS-PAGE analysis of purified EspP derivatives.

DOI: 10.7554/eLife.04234.008

Figure supplement 2. Bam complexes lacking BamB or BamC catalyze the assembly of EspP(46+ $\beta$ ) less effectively than the holocomplex.

DOI: 10.7554/eLife.04234.009

Figure supplement 3. Skp inhibits the assembly of EspP(46+ $\beta)$.

DOI: 10.7554/eLife.04234.010

Figure supplement 4. The efficiency of $\operatorname{EspP}(46+\beta)$ assembly is limited by the ability of the protein to remain folding-competent.

DOI: 10.7554/eLife.04234.011

To obtain additional evidence that EspP derivatives that contain significant passenger domain fragments assemble correctly in the in vitro assay, we selected a few derivatives for further analysis. Initially we found that after incubating $\operatorname{Esp} P(96+\beta)$ with proteoliposomes containing BamABCDE and SurA for $30 \mathrm{~min}$, the cleaved $\beta$ domain was both subject to heat denaturation in SDS and resistant to proteinase K (PK) digestion (Figure 5A). This result provided direct evidence that the $\beta$ domain was not only properly folded, but also inserted into the proteoliposomes. Subsequently we examined the assembly of $\operatorname{EspP}^{\star}(96+\beta)$, a non-cleavable version of $\operatorname{EspP}(96+\beta)$, under the same conditions. In the absence of $\mathrm{PK}$, a band that migrates slower than the folded $\beta$ domain ( $35 \mathrm{kDa}$ vs $\sim 27 \mathrm{kDa}$ ) and that corresponds to the folded form of the full-length protein was observed (Figure 5B). The finding that the $\sim 35 \mathrm{kDa}$ polypeptide was resistant to PK digestion unless detergent was added showed that the passenger domain was translocated into the lumen of the proteoliposomes. We also performed an initial analysis of the assembly of EspP(HA-251+ $\beta$ ), a derivative that contains 251 residues of the EspP passenger domain plus an $\mathrm{N}$-terminal HA tag (14 additional residues). After we incubated the protein with proteoliposomes containing BamABCDE and SurA we detected the $\beta$ domain and the cleaved passenger domain on Western blots probed with anti-EspP C-terminal and anti-HA antisera, respectively, and both fragments were resistant to PK digestion unless detergent was added (Figure 5C). Because the epitope tag is the last segment of the protein that traverses the membrane, these results confirm that the translocation reaction went to completion.

\section{$\beta$ domain assembly is the rate-limiting step in EspP biogenesis}

To examine the biogenesis of EspP derivatives that have different length passenger domains in more detail, we next performed a kinetic analysis of the assembly of EspP $(46+\beta)$ and EspP(HA-251+ $\beta)$. We used $0.2 \mu \mathrm{M}$ substrate in these experiments because we found that doubling the EspP concentration moderately increased the efficiency of assembly. As suggested by a study on OmpT, the enhancement of assembly might be a fortuitous effect of increasing the concentration of urea, which presumably helps to maintain the folding-competence of the substrate (Hagan and Kahne, 2011). Western blot analysis using the anti-EspP C-terminal antiserum showed that $\operatorname{EspP}(46+\beta)$ underwent substantial proteolytic processing within 2 min (Figure 6A, top and Figure 6B, blue curve). Interestingly, EspP(HA$251+\beta$ ) was processed at a very similar rate (Figure 6C, top and Figure 6D, blue curve). Indeed the rate constants obtained by fitting the assembly data for the two EspP derivatives to either a single exponential or two-step model were nearly identical (Supplementary file 1). In the case of the EspP(HA-251+ $\beta$ ) derivative, a PK-resistant $\sim 30 \mathrm{kD}$ polypeptide that corresponds to the cleaved passenger domain accumulated in parallel with the cleaved $\beta$ domain (Figure 6E, top). This observation confirmed that the passenger domain was translocated into the lumen of the proteoliposomes during the assembly reaction. We also introduced a mutation (G1066A) that slightly impairs folding of the $\beta$ domain and thereby delays the initiation of passenger domain translocation by $\sim 1$ min in vivo (Pavlova et al., 2013) into $\operatorname{EspP}(46+\beta)$ and $\operatorname{EspP}(\mathrm{HA}-251+\beta)$. The mutation caused a similar delay in the assembly of both EspP derivatives in vitro (Figure 6 and Supplementary file 1). This observation corroborates the conclusion that defects in the folding of the $\beta$ domain affect the ability of the Bam complex to catalyze subsequent steps in EspP assembly. 


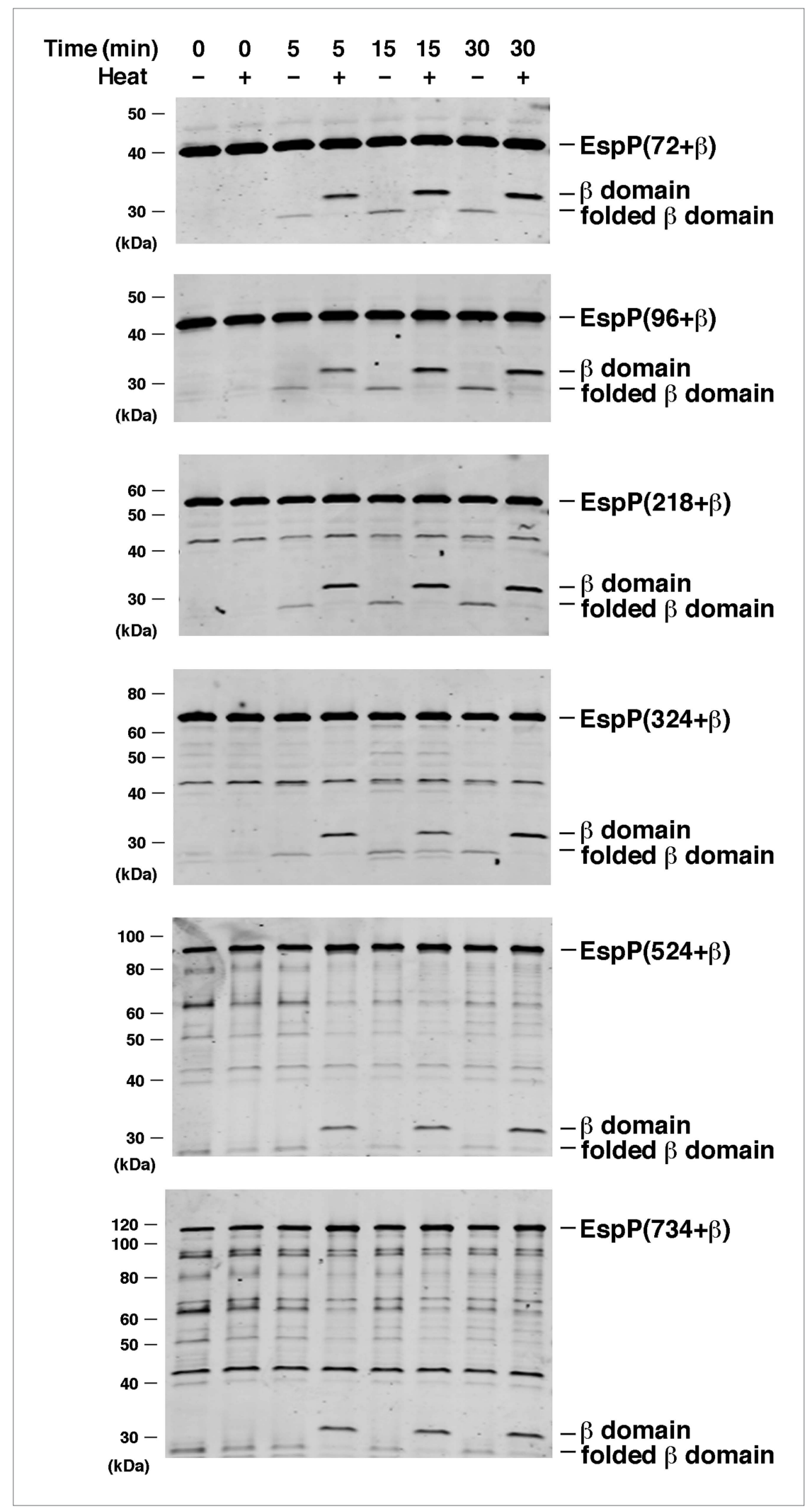

Figure 4. BamABCDE and SurA catalyze the assembly of longer EspP derivatives. The indicated urea-denatured EspP derivative was incubated with SurA and proteoliposomes containing BamABCDE. Aliquots were removed at various time points, heated to $95^{\circ} \mathrm{C}$ or maintained at room temperature after the addition of SDS-PAGE buffer, and Figure 4. Continued on next page 
Figure 4. Continued

analyzed by Western blot using an anti-EspP C-terminal peptide antiserum. The $\sim 27 \mathrm{kDa}$ polypeptide observed at the 0 min time point on the bottom two gels is an unidentified background band.

DOI: 10.7554/eLife.04234.012

Interestingly, we found that an EspP derivative that contains an HA-tagged version of the full-length $\beta$ helix $[\operatorname{EspP}(\mathrm{HA}-714+\beta)]$ was also assembled very rapidly. Despite the presence of a much larger passenger domain, $\mathrm{EspP}(\mathrm{HA}-714+\beta)$ was processed only slightly more slowly than $\mathrm{EspP}(46+\beta)($ Figure $7 A, B)$. Furthermore, the assembly data could be fit equally well to the same kinetic models and did not suggest the existence of an additional slow step (Supplementary file 1). A PK-resistant $\sim 80 \mathrm{kDa}$ polypeptide that corresponds to the cleaved passenger domain accumulated during the assembly reaction (Figure 7 C). As expected, this polypeptide was degraded when the proteoliposomes were solubilized with detergent (Figure 7D). Taken together, the results indicate that passenger domain translocation is relatively fast, and that the assembly of the $\beta$ domain is the rate-limiting step in autotransporter biogenesis. Curiously, the introduction of a short linker into EspP(HA-714+ $\beta)$ that impairs passenger domain folding and stalls translocation in vivo (leva and Bernstein, 2009) only modestly affected assembly in vitro (Figure 7-figure supplement 1). This observation, along with the finding that an EspP chimera containing a $>200$ residue intrinsically disordered passenger domain segment that is secreted efficiently in vivo could also assemble in the in vitro assay (Figure 7-figure supplement 2), suggests that in the absence of an exogenous energy source translocation is not driven exclusively by protein folding.

\section{Assembly of EspP into nanodiscs}

Although it is often assumed that OM protein assembly is catalyzed by a Bam complex monomer, this notion has never been tested. Furthermore, given that autotransporter assembly is inherently more complicated than the assembly of generic $\beta$ barrel proteins, it is conceivable that the Bam complex exists in a distinctive oligomeric state during its interaction with autotransporters. To determine whether a single copy of the Bam complex can mediate the assembly of EspP derivatives, we reconstituted BamABCDE into 10-12 nm nanodiscs using conditions that facilitate the incorporation of a single copy of the $A B C$ transporter $M_{a l F G K}$ (Bao et al., 2012). The nanodiscs eluted at about the same position on gel filtration columns as BamABCDE monomers (Figure 8A). Analysis of the peak fractions by SDS-PAGE suggested that the scaffold protein MSP1D1 was present in excess over the Bam complex proteins (Figure 8B). Because nanodiscs are stabilized by two copies of the scaffold protein (Denisov et al., 2004), however, this observation is consistent with the prediction that they contained a single copy of the Bam complex. In addition, the nanodiscs produced a single band on Blue Native PAGE that migrated only slightly slower than a BamABCDE monomer (compare Figure 8C to Figure $1 \mathrm{C}$ ). Western blot analysis using the anti-EspP C-terminal antiserum revealed that both EspP $(46+\beta)$ and EspP(HA-251+ $\beta)$ were assembled into nanodiscs about as efficiently as they were assembled into proteoliposomes (Figure 8D). Although the non-vesicular nature of nanodiscs precluded assessment of passenger domain translocation using a PK-sensitivity assay, we found that the $\sim 30 \mathrm{kDa}$ passenger domain of $\operatorname{EspP}(\mathrm{HA}-251+\beta)$ accumulated in parallel with the cleaved $\beta$ domain (Figure $8 E$ ). These results not only provide evidence that a Bam complex monomer is sufficient to catalyze autotransporter assembly, but also show that the curvature of the proteoliposome membrane is not itself required for the assembly process.

\section{Discussion}

In this study we identified the minimal set of factors that are required for the complete assembly of the model autotransporter EspP. Initially we showed that the Bam complex reconstituted into proteoliposomes and SurA and both necessary and sufficient to catalyze the folding and proteolytic processing of a simplified version of EspP that consists of the $\beta$ domain plus a short passenger domain fragment that protrudes only slightly from the $\beta$ domain pore. This derivative is similar to generic $\beta$ barrel proteins such as OmpT that reside almost entirely in the $\mathrm{OM}$, except that it contains an internal a-helical segment. In subsequent experiments we found that the same two factors are sufficient to catalyze the translocation of passenger domain fragments associated with much larger EspP derivatives. Interestingly, the efficiency and kinetics of assembly was largely independent of the length of the derivative. While this observation does not exclude the possibility that there are other factors that 


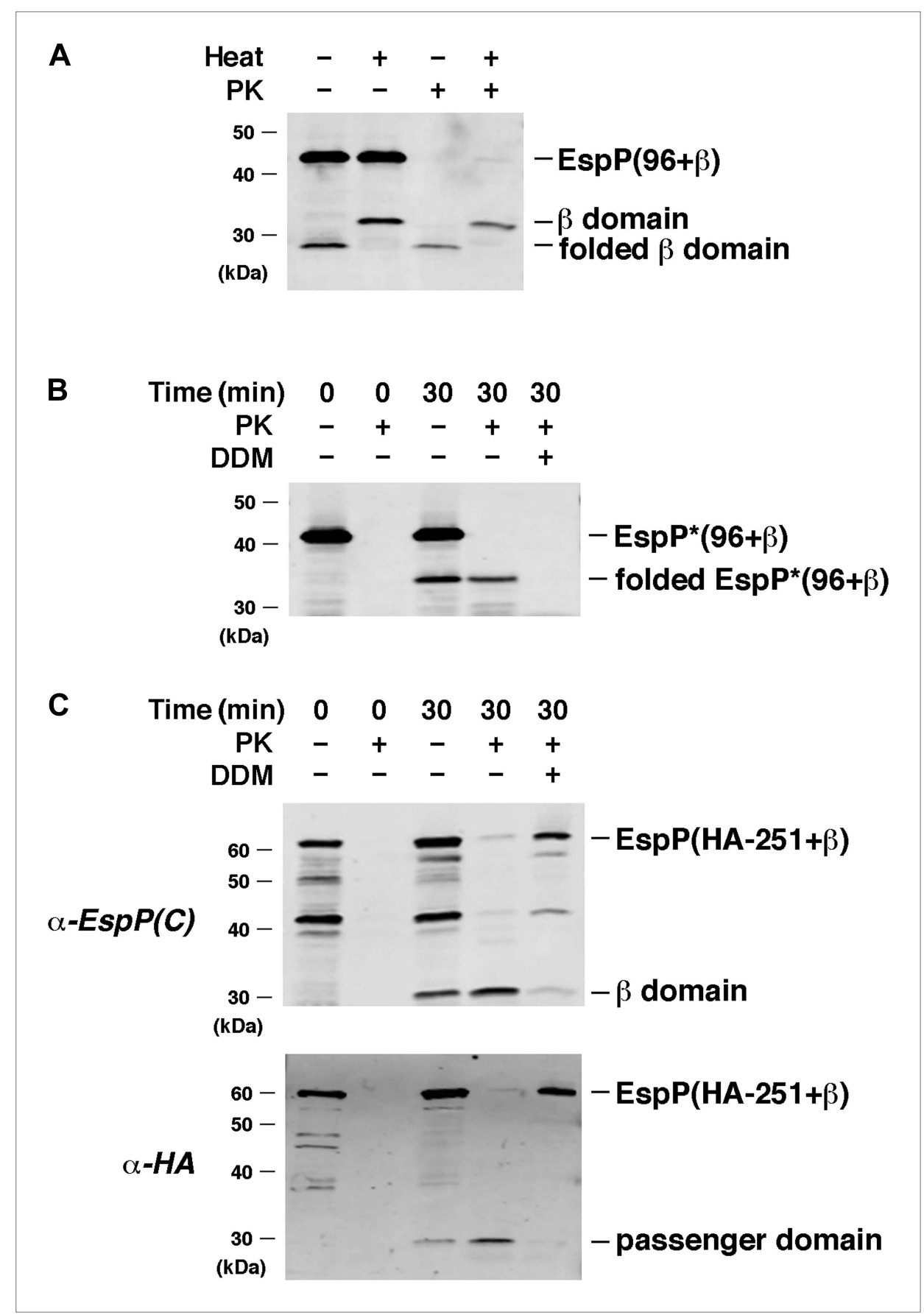

Figure 5. EspP derivatives are correctly assembled into proteoliposomes. (A) Urea-denatured EspP(96+ $\beta$ ) was incubated with SurA and proteoliposomes containing BamABCDE. Aliquots were placed on ice after 0 and 30 min and either treated with PK or left untreated. After the addition of SDS-PAGE buffer samples were heated to $95^{\circ} \mathrm{C}$ or maintained at room temperature and analyzed by Western blot using an anti-EspP C-terminal peptide. (B) The experiment described in (A) was repeated with $\operatorname{EspP}^{*}(96+\beta)$, except that $n$-dodecyl $\beta$-D-maltoside (DDM) was added to one sample prior to PK treatment, and none of the samples were heated after the addition of SDS-PAGE buffer. (C) The experiment described in (A) was repeated with EspP(HA-251+ $\beta$ ), except that DDM was added to one sample prior to PK treatment, and all of the samples were heated to $95^{\circ} \mathrm{C}$ after the addition of SDS-PAGE buffer. Samples were divided in half and analyzed by Western blot using an anti-EspP C-terminal peptide antiserum or an anti-HA antiserum.

DOI: 10.7554/eLife.04234.013 
A

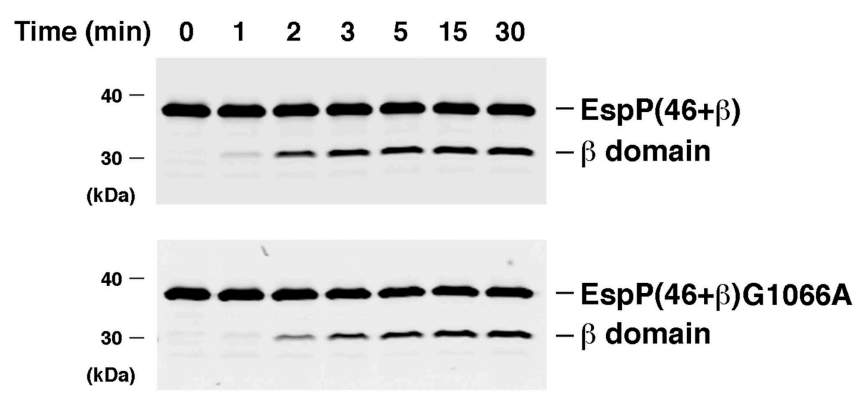

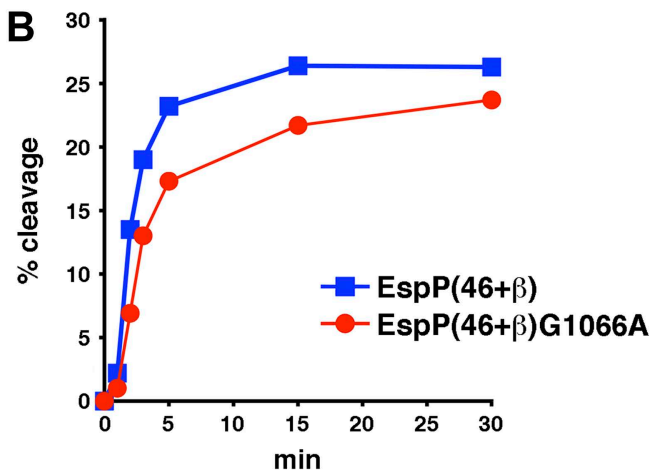

D

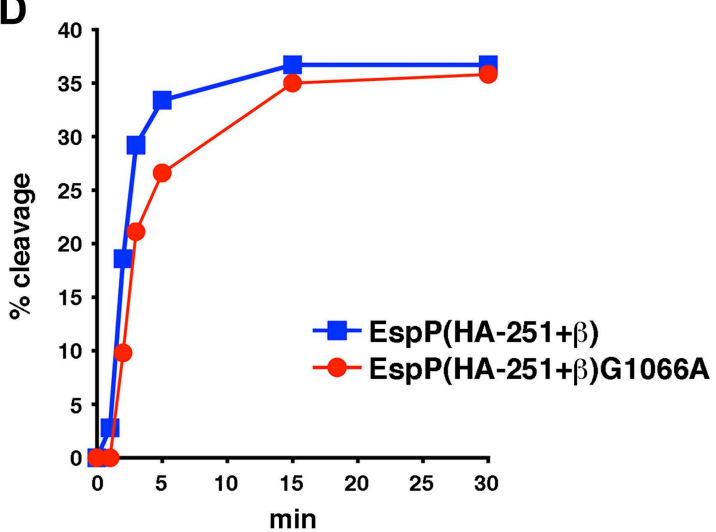

E

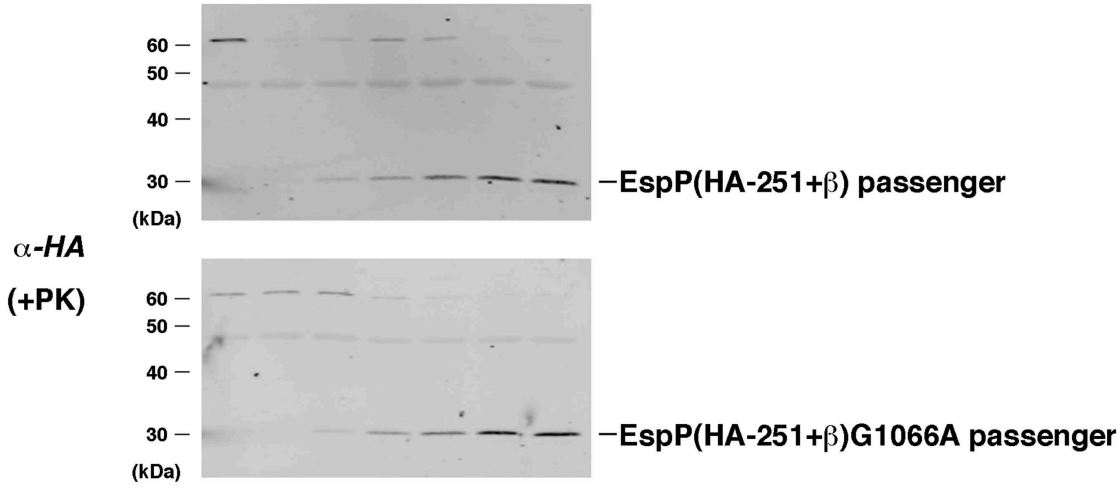

Figure 6. The assembly of the $\beta$ domain is the rate-limiting step in EspP biogenesis. (A) Urea-denatured EspP(46+ $\beta$ ) or EspP(46+ $\beta$ ) G1066A was incubated with SurA and proteoliposomes containing BamABCDE. Aliquots were placed on ice at various time points, heated to $95^{\circ} \mathrm{C}$ after the addition of SDS-PAGE buffer, and analyzed by Western blot using an anti-EspP C-terminal peptide antiserum. (B) Quantitation of the data shown in (A). (C) The

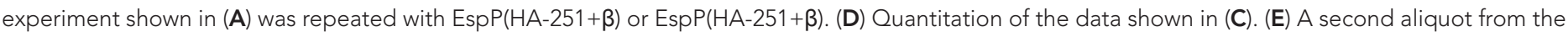
experiment shown in $(\mathbf{C})$ was placed on ice at each time point and treated with PK. After the addition of SDS-PAGE buffer the samples were heated to $95^{\circ} \mathrm{C}$ and analyzed by Western blot using an anti-HA antiserum.

stimulate passenger domain secretion in vivo, it does suggest that they would either play a limited role in the translocation reaction per se or serve to prevent misfolding in the crowded periplasmic space. Finally, experiments using nanodiscs provided evidence that EspP assembly is catalyzed by a single copy of the Bam complex. Overall, our results are consistent with a model in which the passenger domain is secreted through a channel consisting of the open $\beta$ domain and/or the BamA $\beta$ barrel in an open conformation (Figure 1C).

Our work demonstrates that passenger domain secretion does not require either an input of exogenous energy or the presence of IM proteins that transduce energy from the cytoplasm or the 
A
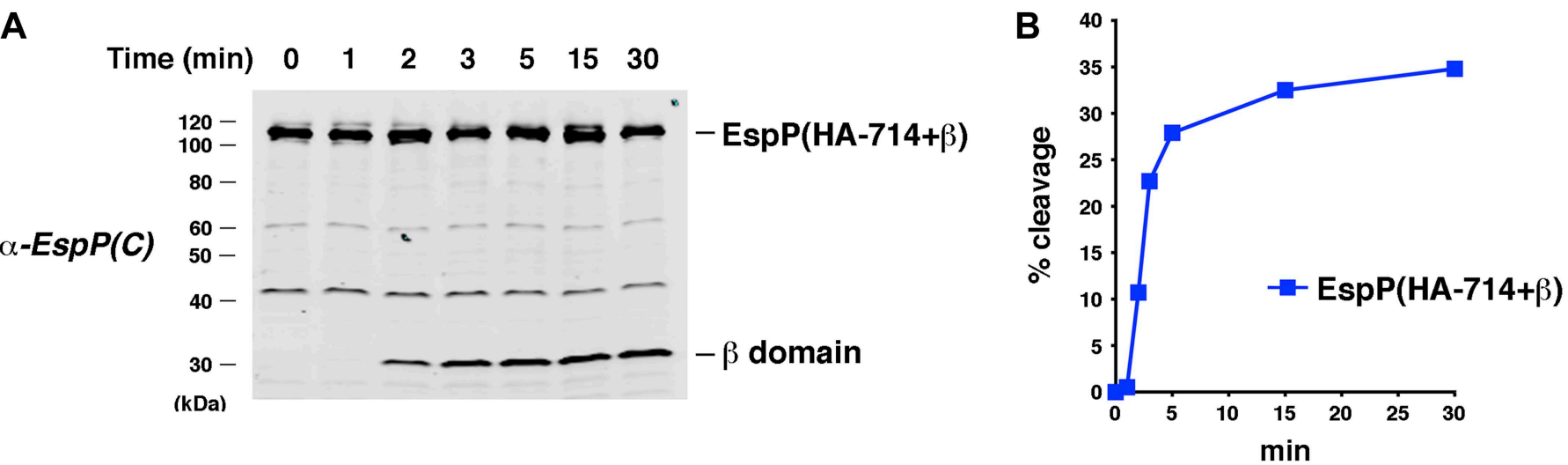

C
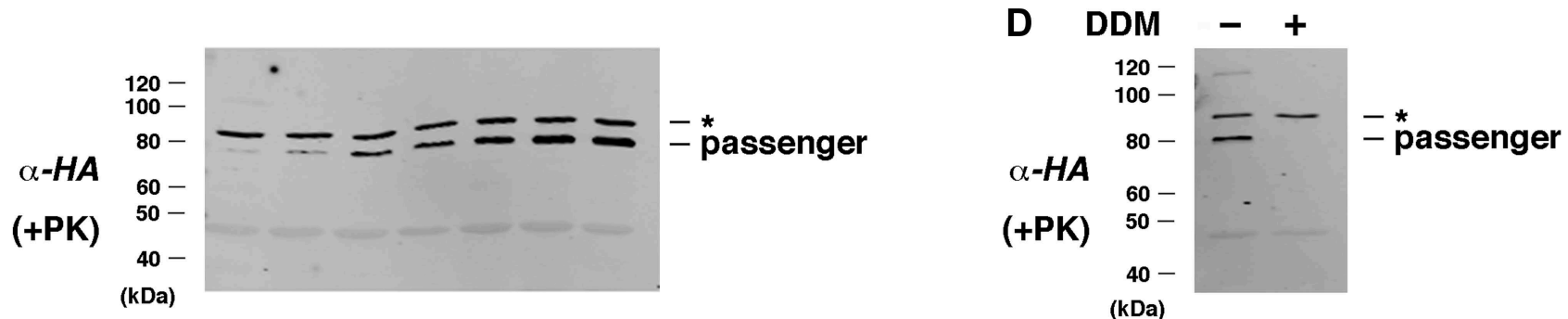

Figure 7. Assembly kinetics of an EspP derivative containing the full-length $\beta$ helix. (A) Urea-denatured EspP(HA-714+ $\beta$ ) was incubated with SurA and proteoliposomes containing BamABCDE. Aliquots were placed on ice at various time points, heated to $95^{\circ} \mathrm{C}$ after the addition of SDS-PAGE buffer, and analyzed by Western blot using an anti-EspP C-terminal peptide antiserum. (B) Quantitation of the data shown in (A). (C) A second aliquot from the experiment shown in (A) was placed on ice at each time point and treated with PK. After the addition of SDS-PAGE buffer the samples were heated to $95^{\circ} \mathrm{C}$ and analyzed by Western blot using an anti-HA antiserum. (D) The experiment described in (A) was repeated. Two equal aliquots were placed on ice after 30 min and DDM was added to one aliquot. The samples were then treated with PK and analyzed by Western blot as described in (C). In (C and D) the asterisk denotes an unidentified background band.

DOI: 10.7554/eLife.04234.015

The following figure supplements are available for figure 7:

Figure supplement 1. Effect of a linker insertion in the passenger domain on the assembly of EspP(HA-714+ $\beta)$. DOI: 10.7554/eLife.04234.016

Figure supplement 2. BamABCDE and SurA catalyze the assembly of RD-EspP chimeras.

DOI: 10.7554/eLife.04234.017

membrane potential. The results are surprising because ATP hydrolysis is required to drive protein translocation through the Sec complex in an analogous reconstituted assay system (Brundage et al., 1990) and because other types of protein translocation reactions appear to require a significant energy expenditure (Alder and Theg, 2003; Shi and Theg, 2013). The high energy cost may result in part from a tendency of proteins to slide backwards at specific stages of the translocation reaction (Schiebel et al., 1991; Bauer et al., 2014). In the autotransporter pathway, the folding of the passenger domain in the extracellular space has been shown to play a role in driving translocation in vivo, and folding may also promote translocation in the in vitro assay. Available evidence, however, neither supports the notion that stepwise folding alone drives translocation nor explains the efficient secretion of the intrinsically disordered RD domain or polypeptides that fold in the periplasm. As previously suggested (Kang'ethe and Bernstein, 2013a), electrostatic or other types of interactions between passenger domains and the Bam complex or membrane lipids may facilitate translocation. Like the FimD protein that secretes type I pilus subunits, the Bam complex might also guide passenger domains along a low-energy pathway (Geibel et al., 2013). Alternatively, the Bam complex might catalyze at least the initial stages of translocation and the assembly of the $\beta$ domain in a concerted fashion. Indeed a model in which a polypeptide segment is pushed across the membrane during the membrane integration of the $\beta$ domain might explain the efficient secretion of $\sim 100$ residue fragments that 
A

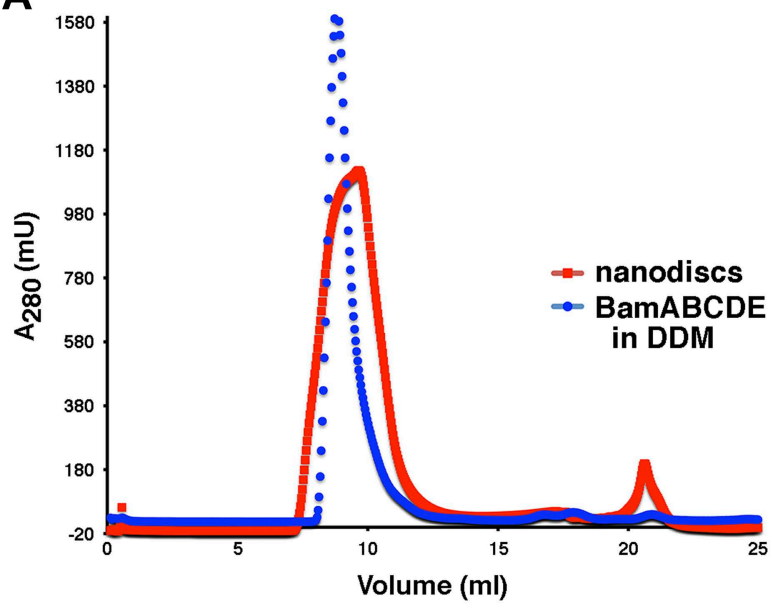

B

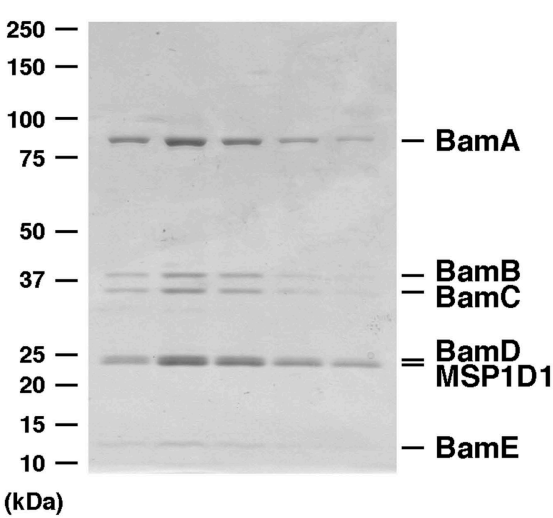

C

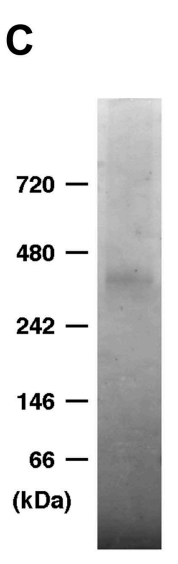

D
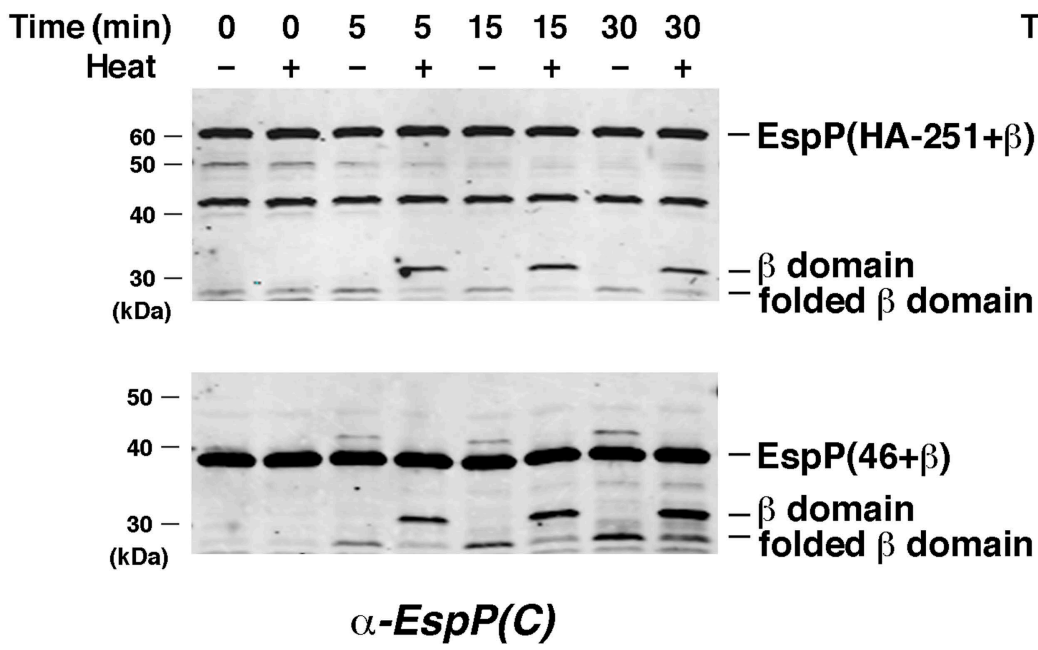

E

Time (min) $\quad 0 \quad 5 \quad 15 \quad 30$

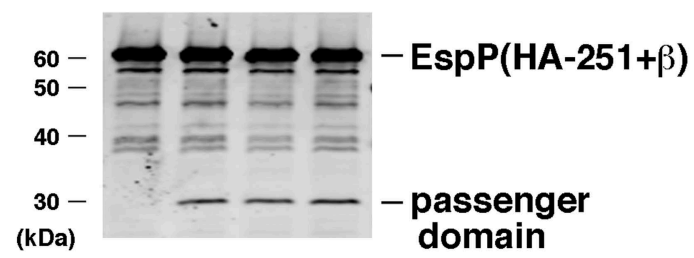

$\alpha-H A$

Figure 8. BamABCDE and SurA catalyze the assembly of EspP derivatives into nanodiscs. (A) Chromatograms of nanodiscs and BamABCDE in DDM on Superdex 75 gel filtration column. (B) SDS-PAGE analysis of the peak fractions in (A). Proteins were visualized by staining the gel with Coomassie Blue. (C) The peak fractions in (B) were pooled and analyzed by Blue Native PAGE. (D) Urea-denatured EspP(HA- $251+\beta)$ or EspP(46+ $\beta$ ) was incubated with SurA and nanodiscs containing BamABCDE. Aliquots were placed on ice at various time points, heated to $95^{\circ} \mathrm{C}$ or maintained at room temperature after the addition of SDS-PAGE buffer, and analyzed by Western blot using an anti-EspP C-terminal peptide antiserum. The $27 \mathrm{kDa}$ polypeptide observed at the 0 min time point on the top gel is an unidentified background band. (E) An additional aliquot from the EspP(HA-251+ $\beta$ ) assembly reaction shown in (D) was removed at each time point, heated to $95^{\circ} \mathrm{C}$ after the addition of SDS-PAGE buffer, and analyzed by Western blot using an anti-HA antiserum.

are too short to fold (Skillman et al., 2005; Pavlova, et al., 2013). While the coupling of translocation to $\beta$ domain assembly might also explain the assembly of a folding-deficient mutant such as EspP(HA$714+\beta) 586$ TEV in vitro, the observation that stalled translocation reactions can be restarted in vivo (leva and Bernstein, 2009; JHP and HDB, unpublished results) strongly suggests that energy can be harnessed after the insertion of the $\beta$ domain is largely complete.

Our results also indicate that the assembly of the $\beta$ domain is the rate-limiting step in autotransporter biogenesis. Pulse-chase labeling and photocrosslinking experiments have previously provided evidence that $\beta$ domain assembly is slower than passenger domain translocation in vivo (leva et al., 2011), but the interpretation of these experiments is complicated by the fact that the synthesis of the 1300 residue EspP protein is itself rather slow ( $45 \mathrm{~s})$. The use of fully synthesized EspP derivatives in the in vitro assay has enabled us to circumvent this problem and assess the contribution of the 
translocation step to the overall reaction kinetics more effectively. It is noteworthy that the ability of the $\beta$ domain to remain assembly-competent also appeared to limit the efficiency of the assembly reaction. This observation corroborates the conclusion that even long passenger domain segments fold slowly and consequently resist aggregation, at least in vitro (Junker et al., 2006). Furthermore, given that the $\beta$ domain clearly interacts with Skp in vivo (leva et al., 2011; Pavlova et al., 2013), it is striking that the presence of the chaperone inhibited assembly in vitro. Presumably we could not recapitulate the productive interaction between Skp and the $\beta$ domain that occurs in vivo, or a factor that is required to release Skp from client proteins was not present (or was present but not fully functional) in our assay.

Finally, the strategy that we have described to purify the Bam complex may facilitate future studies on the assembly of both autotransporters and many other OM proteins. It is striking that, at least in our hands, BamABCDE was more active than Bam(AB)(CDE). While this disparity may simply be due to technical issues, our results also raise the possibility that the structure of the Bam complex assembled in vivo differs from that of the Bam complex reconstructed from BamAB and BamCDE subcomplexes in vitro. Indeed it is conceivable that while a stable heterooligomer can be formed from the two subcomplexes, the Bam complex is actually assembled by a different pathway inside living cells. In this regard it should be noted that in preliminary experiments we obtained evidence that the Bam complex remains intact in vivo and does not undergo a dynamic cycle in which BamAB and BamCDE subcomplexes rapidly dissocate and reassociate (JHP and HDB, unpublished results).

\section{Materials and methods}

\section{Plasmid construction}

Previously described plasmids (Hagan et al., 2010) that encode the BamAB and BamCD subcomplexes, His-tagged BamE, SurA and the His-tagged OmpT G236K/K237G mutant (pSK38, pSK46, pBamE-His, pSK257 and pCH28) were reconstructed. To generate plasmid pJH113, a new Nde I site was introduced into the polylinker of a derivative of PTRC99a that lacks the endogenous Nde I site (Szabady et al., 2005) using the QuikChange Mutagenesis Kit (Agilent, Santa Clara, CA) with the oligonucleotide PTRC/Nde and its complement (all oligonucleotides used in this study are listed in Supplementary file 2). The genes that encode all five subunits of the Bam complex were then cloned sequentially into $\mathrm{pJH} 113$ to create plasmid pJH114. Each gene was amplified by PCR using genomic DNA from E. coli strain AD202 as a template (Akiyama and Ito, 1990). BamA was first cloned into the $\mathrm{Nde}$ I and BamH I sites of pJH113, and the other genes were then cloned into the BamH I site of the resulting plasmid. In the final round of cloning an octahistidine tag was added to the $C$ terminus of BamE during PCR amplification. Plasmids pJH115, pJH116 and pJH117, which encode BamACDE $\left(\mathrm{His}_{8}\right)$, $\operatorname{BamABDE}\left(\mathrm{His}_{8}\right)$, and $\mathrm{BamCDE}\left(\mathrm{His}_{8}\right)$, respectively, were constructed in the same fashion except that one or more of the cloning steps were omitted. Plasmid pJH118, which encodes $\left(\mathrm{His}_{6}\right) \mathrm{BamAB}$, was made by first introducing an Eag I site into pJH113-bamA using oligonucleotide BamA.Eag(+) and its complement. A hexahistidine tag was then introduced at the $\mathrm{N}$-terminus of the mature region of BamA using oligonucleotides BamAHis(+) and BamAHis(-). Finally, a Kpn I-Xba I fragment encoding the $\mathrm{C}$-terminus of BamA and BamB was excised from pJH113-bamAB and cloned into the cognate sites of this plasmid. A plasmid encoding the MSP1D1 protein was previously described (Denisov et al., 2004) and was obtained from Addgene (plasmid 20061).

To make plasmids that express $\mathrm{His}_{6}$-tagged EspP derivatives, fragments of espP were amplified by $\mathrm{PCR}$ using the primer $\operatorname{EspP}(-)$ and an appropriate upstream oligonucleotide and pRLS5, pJH62 or pKMS3 as a template (Skillman et alo, 2005; Szabady et al., 2005). The resulting PCR products were digested with $\mathrm{Nde} \mathrm{I}$ and $\mathrm{BamH} I$ and cloned into the cognate sites of $\mathrm{pET} 28 \mathrm{~b}$. To construct plasmids encoding HA-tagged EspP derivatives, the oligonucleotides HA tag(+) and HA tag(-) (Supplementary file 2) were cloned into the $\mathrm{Nco} I$ and $\mathrm{Nde} I$ sites of plasmids encoding $\operatorname{EspP}(271+\beta)$ and $\operatorname{EspP}(734+\beta)$. To make plasmids expressing RD-EspP chimeras, fragments of the RD gene were amplified using appropriate primers and pWK2 (Kang'ethe and Bernstein, 2013a) as a template. PCR products were then digested with $\mathrm{Nco} I$ and $\mathrm{Nde} I$ and cloned into the cognate sites of pET28b encoding EspP(51+ $\beta)$.

\section{Assembly of the Bam complex from BamAB and BamCDE subcomplexes}

$B a m A B$ and $B a m C D E\left(H_{i s}\right)$ were produced independently essentially as described (Hagan et al., 2010). BL21(DE3) transformed with $\mathrm{pSK} 38$ were grown at $37^{\circ} \mathrm{C}$ in a $1 \mathrm{I}$ volume to $\mathrm{OD}_{600}=0.3$. Cultures were shifted to $25^{\circ} \mathrm{C}$ over $30 \mathrm{~min}$ and bamAB overexpression was induced by the addition of $0.1 \mathrm{mM}$ 
IPTG at $\mathrm{OD}_{600}=0.5-0.6$. Cells were then incubated in the presence of the inducer for $3 \mathrm{hr}$. BL21(DE3) transformed with both pSK46 and pBamE-His were grown at $37^{\circ} \mathrm{C}$ in a $1 \mathrm{I}$ volume to $\mathrm{OD}_{600}=0.5-0.6$. BamCD and bamE $\left(\mathrm{His}_{8}\right)$ expression was induced by the addition of $0.1 \mathrm{mM}$ IPTG, and incubation was continued for an additional 3-4 hr. Cells were pelleted in a Beckman JLA-8.1000 rotor $(4000 \times g$, $15 \mathrm{~min}$ ), resuspended in $10 \mathrm{ml}$ cold $20 \mathrm{mM}$ Tris- $\mathrm{HCl} \mathrm{pH} 8$ and lysed using an Avestin EmulsiFlex $\mathrm{C} 3$ (3-4 passes). Lysates were centrifuged at $5000 \times \mathrm{g}$ at $4^{\circ} \mathrm{C}$ for $10 \mathrm{~min}$ to remove unbroken cells. The supernatants were centrifuged in a Beckman Ti70 rotor $\left(50,000 \mathrm{rpm}, 30 \mathrm{~min}, 4^{\circ} \mathrm{C}\right)$ to pellet the membranes, which were solubilized in $10 \mathrm{ml}$ TBS $(25 \mathrm{mM}$ Tris, $\mathrm{pH} 7.4137 \mathrm{mM} \mathrm{NaCl}, 3 \mathrm{mM} \mathrm{KCl})$ containing $2 \%$ Triton X-100, $10 \mathrm{mM}$ EDTA, and $10 \mu \mathrm{g} / \mathrm{ml}$ lysozyme for $30 \mathrm{~min}$ at room temperature. The ultracentrifugation step was repeated and the resulting supernatants were dialyzed overnight against cold buffer A (TBS/0.5\% Triton X-100). BamAB was then further enriched by gel filtration on an S-200 column (GE Healthcare) equilibrated with buffer $A$. Partially purified BamAB and BamCDE were mixed in at least a 5:1 ratio and rotated in the presence of $2 \mathrm{ml} \mathrm{Ni-NTA}$ agarose for $1 \mathrm{hr}$ at $4^{\circ} \mathrm{C}$. The Ni-NTA beads were washed with one column volume buffer A containing $50 \mathrm{mM}$ imidazole. The assembled Bam(AB)(CDE) complex was eluted with $3.5 \mathrm{ml}$ buffer $A$ containing $500 \mathrm{mM}$ Imidazole and injected into an S-200 column equilibrated with TBS pH 8, 0.03\% n-dodecyl- $\beta$-D-maltoside (DDM), 1 mM tris(2carboxyethyl)phosphine (TCEP). The column was run at $0.5 \mathrm{ml} / \mathrm{min}$ and $1 \mathrm{ml}$ fractions were collected. Portions of each fraction were analyzed by SDS-PAGE to identify fractions that contained complete Bam complexes. Typically 5-7 fractions were pooled and concentrated 10-fold using Amicon Ultra-15 centrifugal filters (Millipore, Billerica, MA). The concentration of the purified Bam complex was then determined using the Bio-Rad DC Protein Assay following the manufacturer's instructions.

\section{Expression and purification of SurA}

His-tagged SurA was overproduced and purified essentially as described (Hagan et al., 2010). In brief, 1 I cultures of $B L 21(D E 3)$ transformed with pSK257 were grown at $37^{\circ} \mathrm{C}$ to $\mathrm{OD}_{600}=1$ and shifted to $16^{\circ} \mathrm{C}$. Cultures were incubated overnight after the addition of $0.1 \mathrm{mM}$ IPTG $(0.1 \mathrm{mM})$. Cells were then harvested as described above, resuspended in $10 \mathrm{ml}$ cold $20 \mathrm{mM}$ Tris $\mathrm{HCl} \mathrm{pH} \mathrm{8}$, and lysed using an EmulsiFlex C3. Lysates were clarified by centrifugation at $35,000 \times g$ at $4^{\circ} \mathrm{C}$ for $20 \mathrm{~min}$. SurA was purified from the resulting supernatant using two consecutive rounds of TALON affinity chromatography (Clontech, Mountain View, CA) following the manufacturer's instructions. The purified protein was dialyzed overnight against $20 \mathrm{mM}$ Tris- $\mathrm{HCl} \mathrm{pH} 8$ to remove the imidazole.

\section{Expression and purification of BamABCDE}

E. coli strain HDB150 (MC4100 ompT::spc $\triangle a r a B A D$ leuD::kan) transformed with pJH114 was grown overnight at $37^{\circ} \mathrm{C}$ in $\mathrm{LB}$ containing $100 \mu \mathrm{g} / \mathrm{ml}$ ampicillin. The cells were washed and diluted 1:80 into $1-2$ I of fresh medium. When the cultures reached $\mathrm{OD}_{600}=0.5-0.6,0.4 \mathrm{mM}$ IPTG was added to induce the expression of bamABCDE. Cells were grown in the presence of the inducer for $1.5 \mathrm{hr}$ and then harvested as described above. Cell pellets were resuspended in $10 \mathrm{ml} / \mathrm{l}$ cold $20 \mathrm{mM}$ Tris- $\mathrm{HCl} \mathrm{pH} 8$ and cells were lysed using an EmulsiFlex C3. Lysates were centrifuged at $6000 \times \mathrm{g}$ at $4^{\circ} \mathrm{C}$ for $10 \mathrm{~min}$. The supernatants were centrifuged in a Ti 70 rotor as described above to isolate total membranes. After the pellets were incubated in $10 \mathrm{ml} / \mathrm{l}$ cold $50 \mathrm{mM}$ Tris pH 8, $150 \mathrm{mM} \mathrm{NaCl}, 1 \%$ DDM on ice for $1 \mathrm{hr}$ the centrifugation step was repeated. Supernatants containing the soluble membrane proteins were then rotated in the presence of $2 \mathrm{ml} / \mathrm{l} \mathrm{Ni-NTA}$ agarose for $1.5 \mathrm{hr}$ at $4^{\circ} \mathrm{C}$. Ni-NTA beads were washed with one column volume buffer $\mathrm{B}(50 \mathrm{mM}$ Tris $\mathrm{pH} 8,150 \mathrm{mM} \mathrm{NaCl}, 0.03 \% \mathrm{DDM})$ containing $50 \mathrm{mM}$ imidazole. BamABCDE was then eluted in $3.5 \mathrm{ml}$ buffer $A$ containing $500 \mathrm{mM}$ imidazole and injected onto a S-200 column equilibrated with buffer $A$. The column was run at $0.5 \mathrm{ml} / \mathrm{min}$ and $1 \mathrm{ml}$ fractions were collected. Fractions that contained complete BamABCDE complexes were identified, pooled and concentrated, and the concentration of the purified protein was determined as described above. NativePAGE (Blue Native) 4-16\% Bis-Tris gels (Life Technologies, Grand Island, NY) were run to verify that the complex was intact. The same protocol was used to produce and purify BamACDE, BamABDE and the BamCDE and BamAB subcomplexes used in Figure 3B and Figure 3-figure supplement 2, except that cells were transformed with pJH115, pJH116, pJH117 or pJH118.

\section{Reconstitution of the Bam complex into proteoliposomes}

E. coli phospholipids (E. coli Polar Lipid Extract, Avanti Polar Lipids, Alabaster, AL) were suspended in water at a concentration of $20 \mathrm{mg} / \mathrm{ml}$ and sonicated until well dispersed. A portion of the phospholipid suspension $(40 \mu \mathrm{l})$ was added to $200 \mu \mathrm{l}$ of the purified Bam(AB)(CDE) or BamABCDE $(20 \mu \mathrm{M})$ and incubated on ice 
for $5 \mathrm{~min}$. The mixture was then diluted with $4 \mathrm{ml}$ of $20 \mathrm{mM}$ Tris $\mathrm{HCl} \mathrm{pH} 8$ and incubated on ice for $30 \mathrm{~min}$ to reduce the detergent concentration and promote proteoliposome formation. The proteoliposomes were pelleted in a Beckman TLA100.4 rotor $\left(50,000 \mathrm{rpm}, 4^{\circ} \mathrm{C}, 30 \mathrm{~min}\right)$ and resuspended in $200 \mu \mathrm{l} 20 \mathrm{mM}$ Tris $\mathrm{HCl} \mathrm{pH}$ 8. Aliquots of the proteoliposomes were flash frozen in liquid nitrogen and stored at $-80^{\circ} \mathrm{C}$.

\section{Expression and purification of MSP1D1}

BL21(DE3) transformed with pMSP1D1 (Denisov et al., 2004) were grown in 1 I cultures at $37^{\circ} \mathrm{C}$, and $0.5 \mathrm{mM}$ IPTG was added at $\mathrm{OD}_{600}=0.7-0.8$. Cultures were incubated for $3 \mathrm{hr}$ after the addition of the inducer. Cells were harvested, resuspended in $10 \mathrm{ml}$ cold $20 \mathrm{mM}$ Tris- $\mathrm{HCl} \mathrm{pH} 8$ and lysed as described above, and lysates were centrifuged at $35,000 \times \mathrm{g}$ at $4^{\circ} \mathrm{C}$ for $20 \mathrm{~min}$. Clarified supernatants were mixed with $2 \mathrm{ml} \mathrm{NiNTA}$ agarose and rotated for $1.5 \mathrm{hr}$ at $4^{\circ} \mathrm{C}$. The beads were washed with one column volume buffer $\mathrm{D}(40 \mathrm{mM}$ Tris $\mathrm{HCl} \mathrm{pH} \mathrm{8,300} \mathrm{mM} \mathrm{NaCl})$ containing $50 \mathrm{mM}$ imidazole. MSP1D1 was then eluted with $5 \mathrm{ml}$ buffer D containing $500 \mathrm{mM}$ imidazole and dialyzed overnight against cold buffer $\mathrm{E}(20 \mathrm{mM}$ Tris $\mathrm{HCl} \mathrm{pH} \mathrm{7.4,100} \mathrm{mM} \mathrm{NaCl}, 0.5 \mathrm{mM}$ EDTA). The purified protein was then flash frozen and stored at $-80^{\circ} \mathrm{C}$. The concentration of MSP1D1 was determined spectrophotometrically using a previously determined $\varepsilon_{280}$ value (http://sligarlab.life.uiuc.edu/nanodisc/protocols.html). Before the protein was used to assemble nanodiscs it was diluted to $0.3 \mathrm{mM}$ in TSGD buffer (50 mM Tris HCl pH $8,100 \mathrm{mM} \mathrm{NaCl}, 0.03 \%$ DDM, 10\% glycerol) (Bao et al., 2012).

\section{Reconstitution of the Bam complex into nanodiscs}

Initially 10-15 ml (dry volume) Bio-Beads (Bio-Beads SM-2 Adsorbent, Bio-Rad, Hercules, CA) were washed and stored at $4^{\circ} \mathrm{C}$ in TS buffer $(50 \mathrm{mM}$ Tris $\mathrm{HCl} \mathrm{pH} 8,50 \mathrm{mM} \mathrm{NaCl})$ as previously described (Bao et al., 2012). Nanodisc reconstitution was performed in a final volume of $300 \mu \mathrm{l}$ essentially as described (Bao et al., 2012) by adding $6 \mu \mathrm{M}$ purified Bam complex, $18 \mu \mathrm{M}$ MSP1D1 and $360 \mu \mathrm{M}$ phospholipids (E. coli Polar Lipid Extract, Avanti Polar Lipids) to TSGD buffer. The mixture was then incubated with Bio-Beads $(50 \mu \mathrm{l})$ overnight at $4^{\circ} \mathrm{C}$ on a rocking platform. The Bio-Beads were then allowed to settle out, and the supernatant was loaded onto a Superdex 75 gel filtration column equilibrated with buffer $\mathrm{F}$ ( $50 \mathrm{mM}$ Tris $\mathrm{HCl}$ pH8, $100 \mathrm{mM} \mathrm{NaCl}, 10 \%$ glycerol). The column was run at a flow rate was $0.5 \mathrm{ml} / \mathrm{min}$, and $0.5 \mathrm{ml}$ fractions were collected. Peak fractions were pooled and concentrated to a volume of $300 \mu \mathrm{l}$ using Amicon Ultra-15 centrifugal filters.

\section{Expression and purification of OM proteins}

OmpT and all EspP derivatives were synthesized without their signal peptides and purified from inclusion bodies. BL21(DE3) transformed with an appropriate plasmid were grown overnight at $37^{\circ} \mathrm{C}$ in $\mathrm{LB}$ containing $50 \mu \mathrm{g} / \mathrm{ml}$ kanamycin and diluted 1:80 into 1 I fresh medium. When the cultures reached $\mathrm{OD}_{600}=0.7,0.5 \mathrm{mM}$ IPTG was added. The cultures were incubated in the presence of the inducer for $3 \mathrm{hr}$. The cells were then harvested, resuspended in $10 \mathrm{ml}$ cold TBS, and lysed as described above. Lysates were centrifuged at $5000 \times \mathrm{g}$ at $4^{\circ} \mathrm{C}$ for $10 \mathrm{~min}$. Pellets containing the inclusion bodies were resuspended in $10 \mathrm{ml}$ TBS and washed twice by repeating the centrifugation step. After the final wash, pellets were resuspended in $5 \mathrm{ml} 8 \mathrm{M}$ urea and incubated at room temperature for $1 \mathrm{hr}$. Samples were then chilled briefly on ice and centrifuged in a Beckman TLA100.4 rotor $\left(26,000 \mathrm{rpm}, 4^{\circ} \mathrm{C}, 20 \mathrm{~min}\right)$. The concentration of the overexpressed OM proteins, which were highly enriched in the supernatants, were determined using the Bio-Rad DC Protein Assay.

\section{OmpT folding assays}

The folding of OmpT was monitored by slightly modifying a previously described assay (Hagan et alo, 2010). OmpT was diluted from a $293 \mu \mathrm{M}$ stock to a final concentration of $20 \mu \mathrm{M}$ and incubated with SurA (final concentration $140 \mu \mathrm{M}$ ) in $50 \mu \mathrm{l} 20 \mathrm{mM}$ Tris pH 6.5 for $10 \mathrm{~min}$ at room temperature. The Bam complex and the fluorogenic peptide Abz-Ala-Arg-Arg-Ala-Tyr $\left(\mathrm{NO}_{2}\right)-\mathrm{NH}_{2}$ (New England Peptide, Gardner, MA) were diluted in $50 \mu \mathrm{l}$ of the same buffer to a final concentration of $16 \mu \mathrm{M}$ and $2 \mathrm{mM}$, respectively. The two sub-reactions were then mixed together and the increase in fluorescence at $30^{\circ} \mathrm{C}$ was monitored on a Spectramax M5 fluorescent plate reader for $1 \mathrm{hr}$ with readings every $20 \mathrm{~s}$. Fluorescence emission was recorded at $430 \mathrm{~nm}$ following excitation at $325 \mathrm{~nm}$.

\section{EspP assembly assays}

An appropriate EspP derivative, SurA and proteoliposomes containing Bam complex were added successively to $20 \mathrm{mM}$ Tris $\mathrm{HCl} \mathrm{pH}$ 8. Reaction components were mixed after each addition. In the 
experiment shown in Figure 3-figure supplement 3, Skp (obtained as a highly purified native trimer from MyBioSource.com, San Diego, CA) was added at the indicated concentration before SurA. In general the final concentration of reaction components was $0.1 \mu \mathrm{M}$ EspP (diluted from a $6 \mu \mathrm{M}$ stock solution in $8 \mathrm{M}$ urea), $1 \mu \mathrm{M}$ SurA and $0.2 \mu \mathrm{M}$ Bam complex. In the experiments shown in Figures 6 and 7 and Figure 7-figure supplement 1, the EspP and SurA concentrations were raised to $0.2 \mu \mathrm{M}$ and $2 \mu \mathrm{M}$, respectively. All assembly reactions were performed at $30^{\circ} \mathrm{C}$. At each time point, aliquots were removed and either mixed with an equal volume of $2 \times$ SDS-PAGE sample buffer or incubated on ice for 5 min with PK $(5 \mu \mathrm{g} / \mathrm{ml})$. In some experiments, 1\% DDM was added prior to PK treatment. Protease digestions were stopped by the addition of $1 \mathrm{mM}$ PMSF and 2× SDS-PAGE sample buffer. To assess the sensitivity of the EspP $\beta$ domain to SDS denaturation, half of each sample was heated at $95^{\circ} \mathrm{C}$ for 5 min while the other half was maintained at room temperature. Proteins were resolved by SDS-PAGE and transferred to nitrocellulose using an iBlot apparatus (Life Technologies). Western blotting was conducted using antisera generated against an EspP C-terminal peptide (Szabady et alı, 2005) or the HA (Y-11) peptide (Santa Cruz Biotechnology, Dallas, TX). Antibody-antigen complexes were detected by incubating filters with an IRDye 680-conjugated goat anti-rabbit antiserum and monitoring fluorescence at $700 \mathrm{nM}$ using an Odyssey infrared imaging system (Licor, Lincoln, NE). Percent substrate cleavage at each time point was defined as $100 \times$ (cleaved $\beta$ domain/unprocessed substrate + cleaved $\beta$ domain).

\section{SDS-PAGE}

Novex $8-16 \%$ minigels (Life Technologies) were used to monitor protein purifications and to resolve the products of all EspP assembly reactions.

\section{Kinetic analysis of EspP assembly}

Data from EspP assembly experiments was fit to single exponential and lag-phase kinetic models using KaleidaGraph 4.1 (Synergy Software, Reading, PA). The percent substrate cleavage at the 30 min time point was defined as maximum assembly and was used to determine a rate constant $\mathrm{k}_{1}$ (or two rate constants $\mathrm{k}_{1}$ and $\mathrm{k}_{2}$ for a sequential two-step model). As in classical enzyme kinetics, $\mathrm{t}_{1 / 2}=\ln 2 / \mathrm{k}$.

\section{Acknowledgements}

We would like to thank Shu-ou Shan for help fitting the data obtained in EspP assembly experiments to kinetic models and Franck Duong for advice on preparing nanodiscs. This work was supported by the Intramural Research Program of the National Institute of Diabetes and Digestive and Kidney Diseases.

\section{Additional information}

Funding

\begin{tabular}{ll} 
Funder & Author \\
\hline National Institute of & Giselle Roman-Hernandez, \\
Diabetes and Digestive & Janine H Peterson, \\
and Kidney Diseases & Harris D Bernstein
\end{tabular}

The funder had no role in study design, data collection and interpretation, or the decision to submit the work for publication.

Author contributions

GR-H, HDB, Conception and design, Acquisition of data, Analysis and interpretation of data, Drafting or revising the article, Contributed unpublished essential data or reagents; JHP, Acquisition of data, Contributed unpublished essential data or reagents

\section{Additional files}

Supplementary files

- Supplementary file 1. Kinetic analysis of EspP assembly in vitro.

DOI: 10.7554/eLife.04234.019

- Supplementary file 2. Oligonucleotides used in this study.

DOI: 10.7554/eLife.04234.020 


\section{References}

Akiyama Y, Ito K. 1990. SecY protein, a membrane-embedded secretion factor of E. coli, is cleaved by the ompT protease in vitro. Biochemical and Biophysical Research Communications 167:711-715. doi: 10.1016/0006291X(90)92083-C.

Alder NN, Theg SM. 2003. Energetics of protein transport across biological membranes. a study of the thylakoid $\Delta \mathrm{pH}$-dependent/cpTat pathway. Cell 112:231-242. doi: 10.1016/S0092-8674(03)00032-1.

Bao H, Duong F, Chan CS. 2012. A step-by-step method for the reconstitution of an ABC transporter into nanodisc lipid particles. Journal of Visualized Experiments e3910. doi: 10.3791/3910.

Barnard TJ, Dautin N, Lukacik P, Bernstein HD, Buchanan SK. 2007. Autotransporter structure reveals intra-barrel cleavage followed by conformational changes. Nature Structural and Molecular Biology 14:1214-1220. doi: 10.1038/ nsmb1322.

Barnard TJ, Gumbart J, Peterson JH, Noinaj N, Easley NC, Dautin N, Kuszak AJ, Tajkhorshid E, Bernstein HD, Buchanan SK. 2012. Molecular basis for the activation of a catalytic asparagine residue in a self-cleaving bacterial autotransporter. Journal of Molecular Biology 415:128-142. doi: 10.1016/j.jmb.2011.10.049.

Bauer BW, Shemesh T, Chen Y, Rapoport TA. 2014. A "push and slide" mechanism allows sequence-insensitive translocation of secretory proteins by the SecA ATPase. Cell 157:1416-1429. doi: 10.1016/j.cell.2014.03.063.

Brundage L, Hendrick JP, Schiebel E, Driessen AJ, Wickner W. 1990. The purified E. coli integral membrane protein SecY/E is sufficient for reconstitution of SecA-dependent precursor protein translocation. Cell 62:649-657. doi: 10.1016/0092-8674(90)90111-0.

Brunder W, Schmidt H, Karch H. 1997. EspP, a novel extracellular serine protease of enterohaemorrhagic Escherichia coli O157:H7 cleaves human coagulation factor V. Molecular Microbiology 24:767-778. doi: 10.1046/j.13652958.1997.3871751.x.

Dautin N, Barnard TJ, Anderson DE, Bernstein HD. 2007. Cleavage of a bacterial autotransporter by an evolutionarily convergent autocatalytic mechanism. EMBO Journal 26:1942-1952. doi: 10.1038/sj.emboj.7601638.

Dautin N, Bernstein HD. 2007. Protein secretion in Gram-negative bacteria via the autotransporter pathway. Annual Review of Microbiology 61:89-112. doi: 10.1146/annurev.micro.61.080706.093233.

Denisov IG, Grinkova YV, Lazarides AA, Sligar S. 2004. Directed self-assembly of monodisperse phospholipid bilayer nanodiscs with controlled size. Journal of the American Chemical Society 126:3477-3487. doi: 10.1021/ ja0393574.

Geibel S, Procko E, Hultgren SJ, Baker D, Waksman G. 2013. Structural and energetic basis of folded-protein transport by the FimD usher. Nature 496:243-246. doi: 10.1038/nature12007.

Hagan CL, Kahne D. 2011. The reconstituted Escherichia coli Bam complex catalyzes multiple rounds of $\beta$-barrel assembly. Biochemistry 50:7444-7446. doi: 10.1021/bi2010784.

Hagan CL, Kim S, Kahne D. 2010. Reconstitution of outer membrane protein assembly from purified components. Science 328:890-892. doi: 10.1126/science.1188919.

Hagan CL, Westwood DB, Kahne D. 2013. bam lipoproteins assemble BamA in vitro. Biochemistry 52:6108-6113. doi: 10.1021/bi400865z.

leva R, Bernstein HD. 2009. Interaction of an autotransporter passenger domain with BamA during its translocation across the bacterial outer membrane. Proceedings of the National Academy of Sciences of USA 106:19120-19125. doi: 10.1073/pnas.0907912106.

leva R, Skillman KM, Bernstein HD. 2008. Incorporation of a polypeptide segment into the $\beta$-domain pore during the assembly of a bacterial autotransporter. Molecular Microbiology 67:188-201. doi: 10.1111/j.13652958.2007.06048.x.

leva R, Tian P, Peterson JH, Bernstein HD. 2011. Sequential and spatially restricted interactions of assembly factors with an autotransporter $\beta$ domain. Proceedings of the National Academy of Sciences of USA 108:E383-E391. doi: 10.1073/pnas.1103827108.

Jong WS, ten Hagen-Jongman CM, den Blaauwen T, Slotboom DJ, Tame JR, Wickström D, de Gier JW, Otto BR, Luirink J. 2007. Limited tolerance towards folded elements during secretion of the autotransporter $\mathrm{Hbp}$. Molecular Microbiology 63:1524-1536. doi: 10.1111/j.1365-2958.2007.05605.x.

Junker M, Besingi RN, Clark PL. 2009. Vectorial transport and folding of an autotransporter virulence protein during outer membrane secretion. Molecular Microbiology 71:1323-1332. doi: 10.1111/j.1365-2958.2009.06607.x.

Junker M, Schuster CC, McDonnell AV, Sorg KA, Finn MC, Berger B, Clark PL. 2006. Pertactin $\beta$-helix folding mechanism suggests common themes for the secretion and folding of autotransporter proteins. Proceedings of the National Academy of Sciences of USA 103:4918-4923. doi: 10.1073/pnas.0507923103.

Kang'ethe W, Bernstein HD. 2013a. Charge-dependent secretion of an intrinsically disordered protein via the autotransporter pathway. Proceedings of the National Academy of Sciences of USA 110:E4246-E4255. doi: 10.1073/pnas.1310345110.

Kang'ethe W, Bernstein HD. 2013b. Stepwise folding of an autotransporter passenger domain is not essential for its secretion. Journal of Biological Chemistry 288:35028-35038. doi: 10.1074/jbc.M113.515635.

Khan S, Mian HS, Sandercock LE, Chirgadze NY, Pai EF. 2011. Crystal structure of the passenger domain of the Escherichia coli autotransporter EspP. Journal of Molecular Biology 413:985-1000. doi: 10.1016/j.jmb. 2011.09.028.

Klauser T, Pohlner J, Meyer TF. 1992. Selective extracellular release of cholera toxin B subunit by Escherichia coli: dissection of Neisseria Iga $\beta$-mediated outer membrane transport. The EMBO Journal 11:2327-2335.

Leyton DL, Rossiter AE, Henderson IR. 2012. From self-sufficiency to dependence: mechanisms and factors important for autotransporter biogenesis. Nature Reviews Microbiology 10:213-225. doi: 10.1038/nrmicro2733. 
Noinaj N, Kuszak AJ, Balusek C, Gumbart JC, Buchanan SK. 2014. Lateral opening and exit pore formation are required for BamA function. Structure 22:1055-1062. doi: 10.1016/j.str.2014.05.008.

Noinaj N, Kuszak AJ, Gumbart JC, Lukacik P, Chang H, Easley NC, Lithgow T, Buchanan SK. 2013. Structural insight into the biogenesis of $\beta$-barrel membrane proteins. Nature 501:385-390. doi: 10.1038/nature12521.

Oomen CJ, van Ulsen P, van Gelder P, Feijen M, Tommassen J, Gros P. 2004. Structure of the translocator domain of a bacterial autotransporter. The EMBO Journal 23:1257-1266. doi: 10.1038/sj.emboj.7600148.

Pavlova O, Peterson JH, leva R, Bernstein HD. 2013. Mechanistic link between $\beta$ barrel assembly and the initiation of autotransporter secretion. Proceedings of the National Academy of Sciences of USA 110:E938-E947. doi: 10.1073/pnas.1219076110.

Peterson JH, Tian P, leva R, Dautin N, Bernstein HD. 2010. Secretion of a bacterial virulence factor is driven by the folding of a C-terminal segment. Proceedings of the National Academy of Sciences of USA 107:17739-17744. doi: 10.1073/pnas.1009491107.

Pohlner J, Halter R, Beyreuther K, Meyer TF. 1987. Gene structure and secretion of Neisseria gonorrhoeae IgA protease. Nature 325:458-462. doi: 10.1038/325458a0.

Renn JP, Junker M, Besingi RN, Braselmann E, Clark PL. 2012. ATP-independent control of autotransporter virulence protein transport via the folding properties of the secreted protein. Chemistry \& Biology 19:287-296. doi: 10.1016/j.chembiol.2011.11.009

Ruiz-Perez F, Henderson IR, Leyton DL, Rossiter AE, Zhang Y, Nataro JP. 2009. Roles of periplasmic chaperone proteins in the biogenesis of serine protease autotransporters of Enterobacteriaceae. Journal of Bacteriology 191:6571-6583. doi: 10.1128/JB.00754-09.

Saurí A, Oreshkova N, Soprova Z, Jong WS, Sani M, Peters PJ, Luirink J, van Ulsen P. 2011. Autotransporter $\beta$-domains have a specific function in protein secretion beyond outer-membrane targeting. Journal of Molecular Biology 412:553-567. doi: 10.1016/j.jmb.2011.07.035.

Sauri A, Soprova Z, Wickström D, de Gier JW, van der Schors RC, Smit AB, Jong WS, Luirink J. 2009. The Bam (Omp85) complex is involved in secretion of the autotransporter haemoglobin protease. Microbiology 155:3982-3991. doi: 10.1099/mic.0.034991-0.

Schiebel E, Driessen AJ, Hartl FU, Wickner W. 1991. $\Delta \mu_{H}+$ and ATP function at different steps of the catalytic cycle of preprotein translocase. Cell 64:927-939. doi: 10.1016/0092-8674(91)90317-R.

Shi LX, Theg SM. 2013. Energetic cost of protein import across the envelope membrane of chloroplasts. Proceedings of the National Academy of Sciences of USA 110:930-935. doi: 10.1073/pnas.1115886110.

Skillman KM, Barnard TJ, Peterson JH, Ghirlando R, Bernstein HD. 2005. Efficient secretion of a folded protein domain by a monomeric bacterial autotransporter. Molecular Microbiology 58:945-958. doi: 10.1111/ j.1365-2958.2005.04885.x.

Szabady RL, Peterson JH, Skillman KM, Bernstein HD. 2005. An unusual signal peptide facilitates late steps in the biogenesis of a bacterial autotransporter. Proceedings of the National Academy of Sciences of USA 102:221-226. doi: 10.1073/pnas.0406055102.

van den Berg B. 2010. Crystal structure of a full-length autotransporter. Journal of Molecular Biology 396:627-633. doi: 10.1016/j.jmb.2009.12.061.

Voulhoux R, Bos MP, Geurtsen J, Mols M, Tommassen J. 2003. Role of a highly conserved bacterial protein in outer membrane protein assembly. Science 299:262-265. doi: 10.1126/science.1078973.

Wu T, Malinverni J, Ruiz N, Kim S, Silhavy TJ, Kahne D. 2005. Identification of a multicomponent complex required for outer membrane biogenesis in Escherichia coli. Cell 121:235-245. doi: 10.1016/j.cell.2005.02.015. Zhai Y, Zhang K, Huo Y, Zhu Y, Zhou Q, Lu J, Black I, Pang X, Roszak AW, Zhang X, Isaacs NW, Sun F. 2011. Autotransporter passenger domain secretion requires a hydrophobic cavity at the extracellular entrance of the ß-domain pore. Biochemical Journal 435:577-587. doi: 10.1042/BJ20101548. 